\title{
Why are there so many bee-orchid species? Adaptive radiation by intraspecific competition for mnemonic pollinators
}

\author{
Michel Baguette ${ }^{1,2}$, Joris Bertrand ${ }^{3}$, Virginie M. Stevens ${ }^{2}$, Bertrand Schatz ${ }^{4}$ \\ ${ }^{1}$ Museum National d'Histoire Naturelle, Institut Systématique, Evolution, Biodiversité, UMR 7205, F-75005 Paris, \\ France
}

${ }^{2}$ Centre National de la Recherche Scientifique and Université Paul Sabatier Toulouse III, SETE Station d'Ecologie Théorique et Expérimentale, UMR 5321, F-09200 Moulis, France

${ }^{3}$ LGDP (Laboratoire Génome et Développement des Plantes) UMR5096, Université de Perpignan Via Domitia CNRS, F-66860 Perpignan, France

${ }^{4}$ CEFE (Centre d'Ecologie Fonctionnelle et Evolutive) UMR 5175, CNRS - Université de Montpellier - Université Paul Valéry - EPHE, 1919 Route de Mende, 34293 Montpellier, France

\section{ABSTRACT}

Adaptive radiations occur mostly in response to environmental variation through the evolution of key eco-morphological innovations that allow emerging species to occupy new ecological niches. However, rapid phenotypic evolution and the evolution of key novelties are likely to also occur when a couple or few species are engaged into narrow ecological interactions. To demonstrate coevolution is a difficult task; only elusive evidences confirm that coevolution is a driver of speciation and diversification. Here we propose that the adaptive radiation of the Mediterranean orchid genus Ophrys, which gave rise to ca. 350 species since the apparition of the genus is due to the particular co-evolutionary dynamics between these plants and their pollinators. We suggest that the pollination by sexual swindle used by Ophrys orchids is the main driver of this coevolution. Flowers of each Ophrys species mimic sexually receptive females of one particular insect species, mainly bees. Male bees are attracted by pseudo-pheromones emitted by Ophrys flowers that are similar to the sexual pheromones of their females. Males lured by the flower shape, color and hairiness attempt to copulate with the flower, which glues pollen on their bodies. Pollen is eventually transferred to the stigma of another flower of the same Ophrys species during similar copulation attempts. Three observations led us to propose the scenario of an asymmetric co-evolutionary relationship between Ophrys and their pollinators. Firstly, there is a strong intra-specific competition among Ophrys individuals for the attraction of their species-specific pollinators, which is due to the high learning and memorization abilities of bees that record the pheromone signatures of kin or of previously courted partner to avoid (further) copulation attempts. Mnemonic pollinators induce thus a strong selective pressure for variation in the pseudo-pheromones emitted by individual flowers, which will potentially generate shifts in pollinator species, and hence Ophrys speciation. These pollinator shifts are adaptive for new Ophrys species because they may benefit from a competitor-free space. Secondly, such shifts in pollinator species are due to the random crossing of peaks in the olfactory landscape of the pollinator guild that is syntopic to each particular Ophrys population. This selective process on individual, random variation in pseudo-pheromone bouquets is followed by directional selection on flower phenotypes that will reinforce the attraction of the new pollinator. Thirdly, pollinators use the pseudo-pheromones emitted by Ophrys to locate suitable habitats from a 
distance within complex landscapes. Pollinators stay fixed for a while in these habitats by the local diversity of pseudo-pheromones, which increases their probability of encounter with a receptive female and hence the reproduction probability of both sexes. Conversely, pollinators disperse out of small suitable habitats once they have memorized the local diversity of sexual pseudo-pheromone bouquet or if fecundated Ophrys flowers repel pollinators, which decreases the probability of geitonogamy (plant advantage) but limit pollinator mating with locally emergent insect females, thus limiting inbreeding and favoring gene flow (pollinator advantage). Finally, we propose several research avenues that emerged according to this scenario of adaptive radiation by assymetric coevolution between Ophrys species and their pollinators.

\section{CONTENTS}

Introduction

1. The actors

1.1 The plant. 5

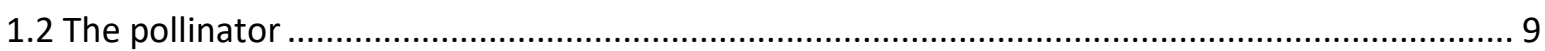

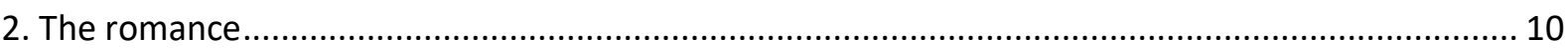

2.1 Pollinator attraction by manipulation of kin and mate recognition mechanisms ...................... 10

2.1.1 Pseudo-pheromones mimic attractive insect female sexual pheromones ........................... 10

2.1.2 Ophrys intraspecific variation in organic compounds ...................................................... 12

2.1.3. Intraspecific competition drives random crossing of peaks in the olfactory landscapes .... 13

2.1.4. Evidences of intra-specific competition and pollinator limitation ...................................... 15

2.1.5. Evolution of floral odor phenotypes and speciation ......................................................... 16

2.1.6 Directional selection of flower shape, colors and hairiness .............................................. 22

2.2 Exploitation of protandry and the resulting directional selection on Ophrys flowering period. 32

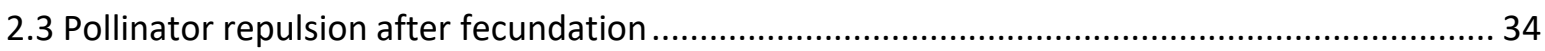

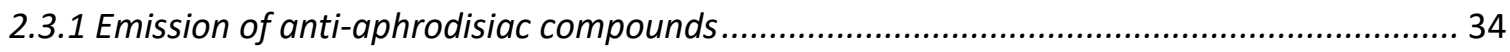

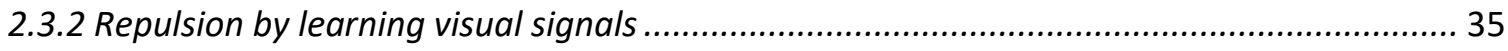

3. The romance from a pollinator viewpoint: repulsion, deception or benefit? .................................. 36

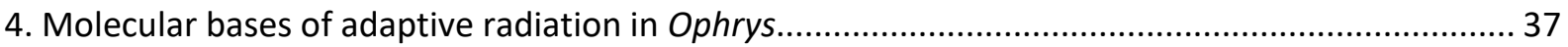

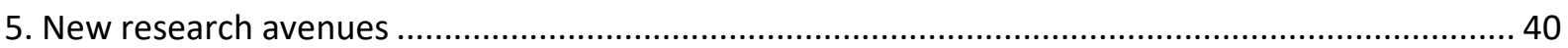

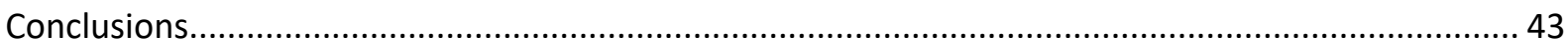

Key words : adaptive radiation, sympatric speciation, pollination by sexual swindle, plant insect coevolution, asymmetric coevolution, chemical ecology, Ophrys orchids, unifying species definition, pseudocopulation, key innovation 


\section{Introduction}

Understanding how and why the diversity of life on earth increased over time is a key research question for biologists (e.g. Hutchinson 1959, Wilson 1992). In the "Origin of species", Darwin (1859) proposed that species evolved according to a speciation/extinction unbalanced process, more species branching on the Tree of Life than those that get pruned from the Tree by extinction. In adaptive radiations, this process is even more unbalanced and radiating groups experience a rapid diversification of species that colonize a variety of ecological niches (Schluter 2000, Gavrilets \& Losos 2009). Recent syntheses proposed that radiations occur mainly through the evolution of key ecomorphological innovations that allow emerging species to occupy new ecological niches (e.g. Simoes et al. 2016, Strouds \& Losos, 2016). Most, if not all of the radiations studied thus far involve the evolution of such key innovations in response to environmental changes.

However, rapid phenotypic evolution and hence the evolution of such key novelties are likely to also occur when a couple or a few species are engaged into narrow ecological interactions (e.g. Grant \& Grant 2006, Litsios et al. 2012). Indeed, when their survivorship depends on the survival of the respective partner(s), species mutually force each other(s) to adapt, which entails coevolution (Solé \& Sardanyés 2014). Coevolution is since long regarded as one of the major processes organizing the earth's biodiversity (Ehrlich \& Raven 1964), even if it is still unclear when and how it may generate species diversity (Thompson 2016). To demonstrate coevolution in the real, natural world is indeed a difficult task (Gomulkiewicz et al. 2007) and so far only elusive evidences confirm that coevolution is a driver of speciation and diversification (Althoff et al. 2014, Hembry et al. 2014; but see Parchman et al. 2006).

This lack of direct evidence of speciation by coevolution contrasts for instance with the preeminent role attributed to the coevolution between plants and their biotic pollinators in the exceptional radiation of Angiosperm species (van der Niet \& Johnson 2012, Schatz et al. 2017), which is presented as the solution to "Darwin's abominable mystery", i.e. the rapid rise and diversification of Angiosperms (Davies et al. 2004). This discrepancy could be due to a more general problem in ecoevolutionary theory that is the prevailing, but often untested conception that ecological and evolutionary processes act on different modes and tempos (Hairston et al. 2005, Schoener 2011). Studies on closely related taxa indeed confirm that their reproductive isolation and hence their speciation (that is, a major evolutionary process) occurred in a few generations only, for instance under the pressure of strong environmental changes (e.g. Lamichhaney et al. 2015, 2016, Parchman et al. 2016). The separation of processes acting on "ecological time" from those that occur in "evolutionary time" may thus be particularly misleading about the role of co-evolutionary processes in speciation, by blurring the fact that adaptations gained by one or several partners involved in coevolution can induce reproductive isolation.

The explosive speciation rate of the Ophrys genus (bee orchids) is among the highest reported in Angiosperms, with values of diversification rates peaking between ca. 4 and 8 lineages million $\mathrm{yr}^{-1}$ in particular clades (Breitkopf et al. 2015). This spectacular radiation gives rise to ca. 350 species in the Mediterranean domain of the western Palearctic (Delforge 2016) since the apparition of the genus ca. $4.910^{6} \mathrm{yr}$ ago (Breitkopf et al. 2015). Here we propose that this spectacular adaptive radiation is due to the particular co-evolutionary dynamics between these plants and their pollinators. We suggest that the unusual pollination mechanisms used by bee orchids is the main driver of this coevolution. Bee orchids use pollination by sexual swindle: flowers of each Ophrys 
species mimic sexually receptive females of one particular species of insects, mainly solitary bees (Hymenopterans). Males of the corresponding insect species are lured by efficient sexual stimuli produced by the flowers (e.g. Schiestl et al. 1999). They are attracted by a blend of organic compounds emitted by Ophrys flowers that mimic the sexual pheromone odor bouquet of their females (pseudo-pheromones). The phenotypes of the flowers induce male landing and pseudocopulations, i.e. copulation attempts of the insect males with the flower. Pollen is glued on male's body (generally on his head or his abdomen) by his frenetic movements on the flower, and eventually transferred to the stigmatic cavity of another flower of the same Ophrys species during similar copulation attempts.

Pollination by sexual swindle is the most specialized pollination strategy in orchids (Scopece et al. 2007). Evolutionary specialization in plant-pollinator interactions refers to the process or trend of evolving from ecologically or phenotypically less specialized to more specialized (Armbruster 2017). However, symmetric coevolution in which the evolution of flowers towards more specialized pollination, and the evolution of animals towards more specialized use of floral resources occur concurrently is rare (Armbruster 2017). Here we propose an asymmetric co-evolutionary relationship between Ophrys and their pollinators, in which plants strictly rely on insects for their reproduction, whereas pollinator rewards are male (re)location in suitable habitats with benefits on male and female reproductive success and hence consequences on insect population spatial dynamics.

Our scenario of asymmetric co-evolutionary dynamics between Ophrys and their pollinators relies on three observations:

1. There is a strong intra-specific competition among Ophrys individuals for the attraction of their species-specific pollinators, which is due to the high learning and memorization abilities of these Hymenopterans that record the pheromone signatures of kin or of previously courted partner to avoid (further) copulation attempts. Pollinator limitation is widely observed in Angiosperms (e.g. Van der Niet et al. 2014), which is here exacerbated by the refined cognitive processes of most Ophrys pollinators. Mnemonic pollinators induce thus a strong selective pressure for variation in the pseudo-pheromone bouquets emitted by the flowers, which will potentially generate shifts in pollinator species, and hence Ophrys speciation. These pollinator shifts are adaptive for new Ophrys species because they may benefit from a competitor-free space. Such shifts are facilitated by the availability of a large number of potential pollinator species. Indeed, contrarily to most Angiosperms, the diversification and the radiation of Ophrys began and occurred in a world in which Insects and more specifically Hymenopterans were already highly diversified (Condamine et al. 2016).

2. Such shifts in pollinator species are thus due to the random crossing of peaks in the olfactory landscape (Svensson et al. 2014) of the pollinator guild that is syntopic to each particular Ophrys population. This selective process on individual, random variation in pseudopheromone bouquets would then be followed by directional selection on flower phenotypes and on flowering period that will reinforce the attraction of the new pollinator and hence the reproductive success of the new Ophrys species.

3. Pollinators use the pseudo-pheromone bouquets emitted by Ophrys to locate suitable habitats from a distance within complex landscapes. Pollinators stay fixed for a while in these 
habitats by the local diversity of pseudo-pheromone bouquets, which may increase their probability of encounter with a receptive female and hence the reproduction probability of both sexes. Conversely, pollinators may disperse out of small suitable habitats once they have memorized all the local diversity of sexual pseudo-pheromone bouquet or if fecundated Ophrys flowers repel pollinators, which decreases the probability of geitonogamy (plant advantage) but limit pollinator mating with locally emergent insect females, and hence limit inbreeding and favors gene flow (pollinator advantage).

We will present briefly some general elements of the biology of the actors i.e. the Ophrys species and their pollinators that are relevant to the observations mentioned above. Next, we will review their romance, i.e. the interspecific interactions leading to pollination by sexual swindle, in the context of intra-specific competition between individual plants for mnemonic and limited pollinators. Then we will discuss if and how this intra-specific competition could fuel speciation in sympatry and the rapid radiation in Ophrys. Finally, we will propose some critical research avenues issued from this review.

\section{The actors \\ 1.1 The plant}

Ophrys is a monophyletic genus of orchids that are endemic of the western Palearctic mainly in the Mediterranean region (Devillers \& Devillers-Terschuren 1994, Delforge 2016). The Mediterranean region forms a band that covers ca. $4000 \mathrm{~km}$ in latitude, and is one of the largest archipelagos in the world with five main islands and about 10,000 islands and islets (with approximatively only 250 inhabited by humans). This geographic situation combined to a high geological diversity, to a complex climatic history during the glaciation - deglaciation cycles over the Quaternary inducing sea level variation and land uplift and subsidence, to fire disturbances and to the long-lasting influence of humans even before the Antiquity shaped a mosaic of landscapes offering a wide variety of environmental conditions (Blondel \& Aronson 1999). Most Ophrys species occur in mesic to dry grasslands, shrublands or light woodlands where the cover and height of the herbaceous layer are limited, and hence the interspecific competition with other herbaceous species is rather low. The spatial distribution of Ophrys populations maps thus onto the distribution of such habitats, and is generally discontinuous in the complex Mediterranean landscapes according to edaphic and mesoclimatic conditions, fire regimes or human land use.

Ophrys are allogamous ${ }^{1}$ long-lived plants (up to $20 \mathrm{yr}$ ) that flower during 4-6 weeks once a year, and each individual plant does not necessarily flower each year (Wells \& Cox 1991, Hutchings 2010). Ophrys stems carry as far as 15 flowers that open more or less successively (Delforge 2016). Current knowledge of Ophrys biology indicates that all species but two ${ }^{2}$ use pollination by sexual swindle (Delforge 2016). In this particular pollination syndrome, three different stimuli acting successively in space and time to attract a male insect to an Ophrys flower (e.g. Fransisco \& Ascensao 2013). The flowers emit an odor bouquet of volatile organic compounds similar to the sexual pheromones produced by virgin females of the pollinator species (pseudo-pheromones) that attract the male at a distance of ca. 5-10 m (Paulus 2006). Together with the emission of pseudopheromones, the shape and the color of the flower induce the male pollinator landing on the flower

\footnotetext{
${ }^{1}$ With the exception of Ophrys apifera that can be partially autogamous, which explains its occurrence and reproduction outside the geographical range of its Hymenopteran pollinator.

${ }^{2}$ Ophrys apifera, as already mentioned is partially autogamous, whereas Ophrys helenae provides shelters to at least two species of solitary bees (Verrecken et al. 2012).
} 
(e.g. Rakosy et al. 2012, 2017, Paulus 2018). The shape (Rakozy et al. 2017) and the hairiness (Ågren et al. 1984) of the flower orient the body of the male in parallel to the longer length of the labellum on the flower. The pollinator landing on an Ophrys labellum may detect by antennal contact pseudopheromones emitted by the flower that coincides with the odor of a new, potential sexual partner, which will initiate his copulation behavior. According to Ophrys clades, the emission of pseudopheromones is concentrated either on the top of the labellum or on its bottom, which explained that the part of the insect's body close to the anthers where the pollen will be glued is either its head or its abdomen. The male will extend his genital apparatus and poke the labellum with his abdomen. During these movements, either his head or his abdomen will come into contact with sticky, coherent masses of pollen grains that will get glued totally or partially on these body parts (Kullenberg \& Bergström 1976).

Like in other orchid species, pollen grains of Ophrys are grouped in massulae, which correspond to a coherent mass of pollen grains developed from a single pollen mother cell. Massulae themselves are grouped into pollinia; there are two pollinia per Ophrys flower (Claessens \& Kleynen, 2011, 2016). The copulation attempt ends up typically after 10 to 30 seconds by the male takeoff. He may be attracted to another conspecific flower where this behavioral sequence is repeated. This new copulation attempt will eventually lead to the deposit of either massulae or of the entire pollinia on the stigmatic cavity of the flower (Paulus 2006). After its capture by a pollinator the pollinia bends forwards, which will favor the physical contact with the stigmatic cavity of another flower (Johnson \& Nilsson, 1999, Claessens and Kleynen, 2011, 2016).

Each fecundated flower will produce thousands of tiny seeds (e.g. Nazarov \& Gerlach 1997, Paulus 2006) that are wind transported (e.g. Salisbury 1975). Seeds are so small that they have no nutrient reserves. They will require interactions with fungi and need to form mycorrhiza to germinate. The presence of suitable fungi species that are required to establish mycorrhiza can be thus key for Ophrys species establishment, like in other orchids (e.g. McCormick \& Jacquemyn 2014, Jacquemyn et al. 2015, Rasmussen et al. 2015). A couple of phylogenetically close species shared mycorrhizal partners in sympatry however, suggesting little or no importance of mycorrhizal symbiosis in reproductive isolation (Gervasi et al. 2017), which is also observed in other orchid genera (Schatz et al. 2010). The generation time from a seed to a reproductive plant is still not known precisely, but according to observation of seedling development, it should take two to several years (Fabre 1852, Hutchings 2010, Jaquemyn \& Hutchings 2015).

The species richness within the Ophrys genus varies from 9 to 354 species in the literature (summarized in Tyteca \& Baguette 2017, Bateman 2018, Bateman et al. 2018a) according to the species' definition used by systematic authorities. Admittedly, there is still a harsh debate around the biological meaningful species' definition to use within Ophrys (e.g. Bateman et al. 2011, Vereecken et al. 2011, Bateman 2018), which led to the statement that there is a taxonomic exaggeration in European orchids (the "orchid fever") due to the pre-eminence of splitters over lumpers among European taxonomists (Pillon \& Chase 2007). Although we acknowledge that such taxonomic exaggeration exists, we believe that there are increasing evidences to confidently support the reproductive isolation, evolutionary divergence and hence species formation in several hundreds of Ophrys taxa. 
Here we use the unifying species definition coined by de Queiroz $(2005,2007)$ that considers species as separately evolving metapopulation lineages. This definition was initially applied to the genus Ophrys by Devillers \& Devillers-Terschuren (2013), who focused on the evolution of those phenotypic characters that are targets of selective pressures eventually leading to speciation, and hence that will serve as diagnosis of species delimitation. In this paper, we show that the unifying species definition is also operational to understand the emergence, the establishment and the maintenance of such characters leading to the differentiation of metapopulation lineages, and hence to speciation, in Ophrys. Metapopulations are groups of local populations that are connected by dispersal (e.g. Hanksi 1999). Within a landscape, individuals of a species are restricted to areas of habitat and form thus local populations where there are suitable conditions for them to complete their life cycle. This includes for Ophrys both above (light, wind and pollinators) and below ground $(\mathrm{pH}$, nutriments and fungi) elements. Therefore, for Ophrys individuals, the landscape may be viewed as a mosaic of suitable habitat patches with or without local populations embedded within an area of unsuitable habitat, or matrix. If male gametes (pollen) or zygotes (seeds) leave their current habitat they have to disperse across the matrix and are therefore exposed to risks that may result in death. However, the dispersing male gametes or zygotes may have the reward of arriving at another habitat patch with suitable above and below ground conditions (and for gametes, with receptive flowering individuals). The overall result of this dispersal is that local populations are not isolated but are in fact connected.

Metapopulations have properties that the local populations do not possess alone. Firstly, the persistence of metapopulations is more stable than that of local because population extinctions can be counter-balanced by the creation of new ones (colonization) elsewhere in the landscape following successful dispersal events. Secondly, the dynamic nature of a metapopulation (that is, gene flow associated with dispersal and local population turnover) contributes to its genetic structure and diversity, and hence to its evolutionary trajectory (e.g. Baguette et al. 2017). In Ophrys metapopulations, wind dispersal of seeds performed (re)colonization, whereas gene flow among local populations occurs both through seed dispersal and through the dispersal of pollinia carried by pollinators among habitats patches within landscapes. The complexity and the time scale of these two types of dispersal are dramatically different, however: several years may elapse between the landing of a seed and its first flowering, whereas gene flow associated to pollinia transfers occur on a yearly basis. Several studies reveal unambiguously that the reproductive success of Ophrys individual plants is low to very low (e.g. Neiland \& Wilcock, 1998, Claessens \& Kleynen 2011), and three studies report that this crucial fitness parameter depends significantly on their population spatial structure (Vandewoestijne et al. 2009, Gervasi et al. 2017, Borràs \& Cursach 2018). Considering Ophrys species as separately evolving metapopulation lineages has thus the immense merit of putting the evolutionary dynamics processes associated to pollination by sexual swindle in their spatio-temporal contexts.

According to the unifying species concept, the pre-zygotic isolation mechanism associated with the attraction of a specific pollinator species appears to be a sufficient criterion to delineate an Ophrys species (Paulus 2006, 2018, Paulus \& Gack 1990, Vereecken et al. 2011, Breitkopf et al. 2015). Leading Ophrys systematists concur with this view by stressing that in this genus, the complex of adaptations leading to the attraction of a specific pollinator within populations of the same lineage is the main norm to delimit a species (Devillers \& Devillers-Terschuren 2013). The integrative taxonomy approach (Dayrat 2005; Padial et al., 2010) integrates a wider array of biological characters than 
pollinator identity only (e.g. plant and flower morphology and phenology, population genetic structure, ecological preferences). This approachusually confirms species diagnosis by the attraction of a specific pollinator species, and hence validates the use of the unifying species definition (Joffard et al., 2016; Joffard et al. submitted).

On this basis, we consider that the genus includes ca. 350 species in the Western Palearctic (Delforge 2016), which are individualized by the convergence of both pollinator identities and plant phenotypic characters, rather than 9-11 species separated by their genome dissimilarities (Bateman 2018, Bateman et al. 2018a), which rather correspond to 9 to 11 different clades. The use of DNA sequences to infer Ophrys systematics is currently a conundrum; we just mention here that the more informative study is a time-calibrated phylogeny based on the analysis of the sequences of 6 nuclear loci in 37 Ophrys species by Breitkopf et al. (2015), which showed that the diversification rate of Ophrys species was not constant over time. Overall, this phylogeny is consistent with the results of Bateman et al. (2018a), which is based on 34 accessions and a GBS protocol with 4159 SNPs. Since the putative origin of the group (ca. $4.910^{6} \mathrm{yr}$ ago), the radiation of the Ophrys genus seems to depend on an increase in the diversification rates of some particular clades, which was related to possible pollinator shifts within these clades (Breitkopf et al. 2015, Figure 1). However, how and why such shifts do occur still remain open questions.

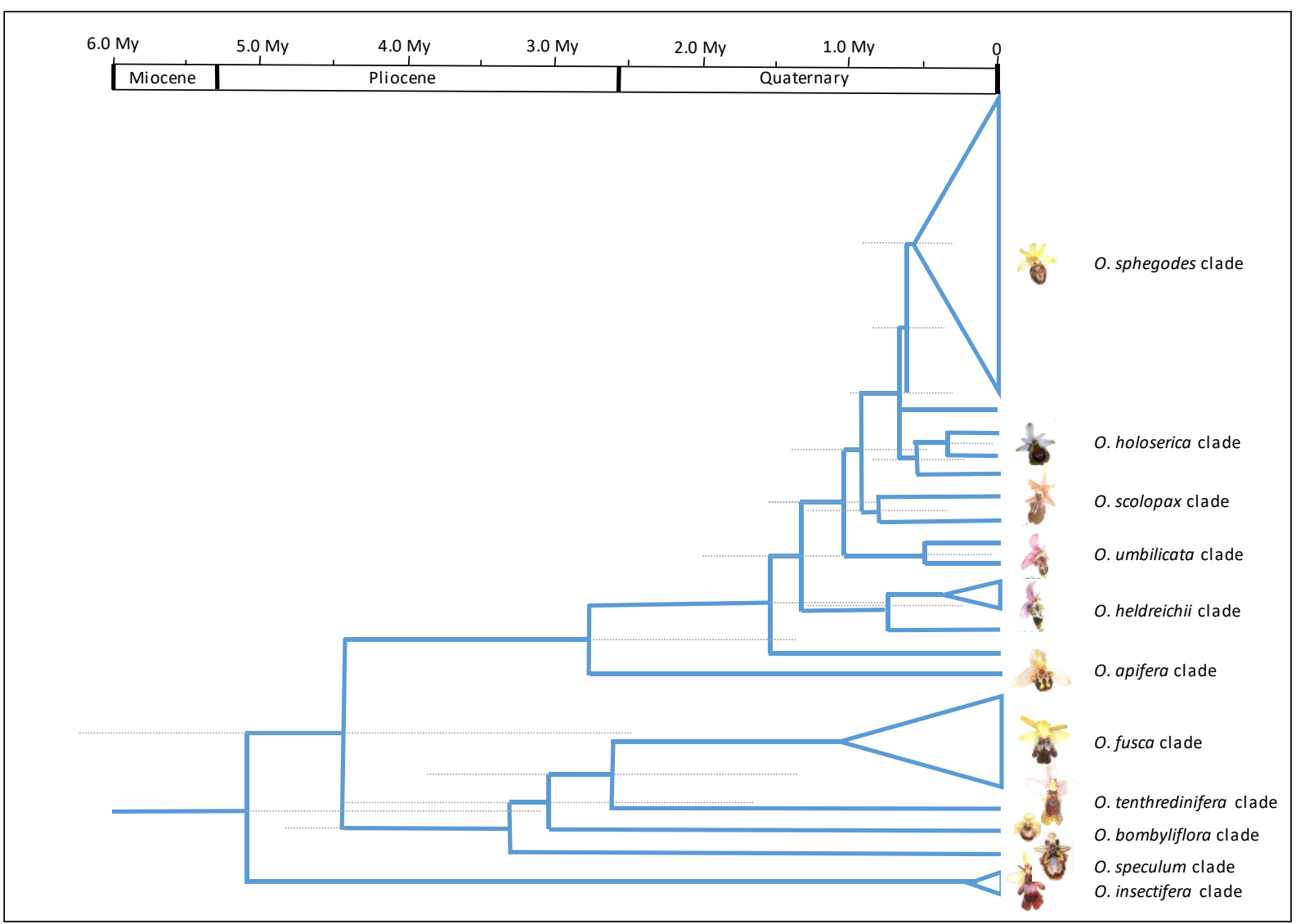

Figure 1. Extreme adaptive radiation in bee-orchids (Ophrys). In this group, more than 350 species evolved around the Mediterranean basin over less than 6 million of years, a diversification rate almost unrivaled worldwide. The figure illustrates the time calibrated (in million years) phylogenetic relationships between 11 significantly different clades inferred from DNA sequences from 37 species and their floral phenotypes. Triangles depict rapid ongoing radiations in the corresponding clade; the diversification rate in these clades is higher than the mean diversification rate in the genus (modified from Breitkopf et al. 2015, Open Access). Interestingly, there are two phases of diversification since there are 
only three clades up to $3.410^{6} \mathrm{yr}$ and seven clades up to $2.510^{6} \mathrm{yr}$ before a more rapid diversification leading to the present situation with 11 clades (Figure 1). Like all others, this molecular phylogeny confirms the monophyly of the genus Ophrys, and invalidates its division in two sub-genus grouping species with either abdominal (Pseudophrys, basal) or cephalic (Euophrys, derived) position of the pollinator on the plant during copulation attempts.

\subsection{The pollinator}

Most currently known pollinators of Ophrys (>98\%) are males of Aculeata (Hymenoptera) species belonging to several families of solitary bees (Apidae, Megachilidae, Colletidae, Halictidae and Andrenidae). Exceptions are males of two species of Crabronidae (Hymenoptera Aculeata), of one species of sawfly (Hymenoptera Symphita), of two species of scarabeid beetles (Coleoptera Scarabeidae) and of one species of colonial bees (Bombus, Hymenoptera, Apidae) (Classens \& Kleynen, 2011, 2016, Gaskett 2011, Delforge 2016, Paulus 2018).

The taxonomy of the ca. 2000 wild bee species in Europe was recently reviewed (e.g. Danforth et al. 2013, Fortel et al. 2014). Solitary bees are particularly diversified and abundant within the Mediteranean region, in habitats similar to those described above as suitable for Ophrys species (Rasmont \& Haubruge 2014). In solitary bees, the adult stage usually lasts for only three or four weeks, whereas some species have two or even three adult generations a year. During their brief adult life, males will patrol around nesting areas or rendez-vous sites hoping to mate with a female (Paxton 2005). Females of most species will mate only once soon after their emergence from pupae they store the sperm and release it when needed - and then spend their time creating and provisioning a nest in which to lay their eggs. Female solitary bees have amazing engineering skills, and go to extraordinary lengths to construct a secure nest, as carefully documented by skilled natural historians (Ferton 1923, Fabre 1924). In natural conditions, solitary bees will nest in all sorts of places. Most species, however, nest in the ground, digging a tunnel in bare or partially vegetated, welldrained soil or use abandoned beetle burrows or other tunnels in snags.

The mode of sex determination in these Hymenopterans is haplodiploïdy associated with a complementary sex determination locus (Heimpel \& De Boer 2008). In haplodiploidy, fertilized eggs develop as females and unfertilized eggs as males. As males emerge from unfertilized eggs, this mechanism prevents transfer of information from a father to its offspring. However, haplodiploïdy associated with a single locus complementary sex determination renders Hymenopterans highly sensitive to inbreeding, which in addition to its various deleterious effects (e.g. Charlesworth \& Charlesworth 1987), translates into the production of diploid, infertile males in this group (e.g. Zayed \& Packer 2005). Hymenopterans have thus developed three mechanisms of inbreeding avoidance: (1) kin recognition (recognition and avoidance of kin as mates, e.g. Pusey \& Wolf 1996), (2) protandry (adult males emerge from their pupae and are active one week or two weeks before females, e.g. Eickwort \& Ginsberg 1980) and (3) dispersal (one sex, here male disperses more often and further than the other, Gandon 1999, Perrin \& Mazarov 1999). Maximum dispersal distances recorded in male bees related to Ophrys pollinators were around $2.5 \mathrm{~km}$ (Dos Santos et al. 2016), with most of them having a foraging distance of several hundreds of meters.

Behavioral observations of Hymenopterans showed that mating with kin is avoided by the emission by both sexes of an individual odor signature (Ayasse et al. 2001). Laboratory and field studies of solitary bees show that females produce individually distinctive sexual pheromones that are attractive to males (Wcislo 1987, 1992). There is considerable inter-individual variation in female attractiveness to males among sexually immature females (Wcislo 1987, Wcislo 1992), and males 
from the same population had a striking consistency towards female's attractiveness (Wcislo 1987). Moreover, males used sexual pheromone signals to learn the identity of female bees with which they have attempted to copulate, thereby avoiding these females in further encounters (Smith and Ayasse, 1987, Wcislo 1987, 1992). Also, the fragrance proper to each female has the function to inform about their receptivity. Altogether, female olfactory "identity cards" allow males to save time by not trying to copulate with non-receptive individuals (Barrow et al. 1975, Wcislo 1987, 1992).

The chemical basis of individual identity recognition in Hymenopterans has been worked out for the solitary bee Colletes cunicularius (Vereecken et al. 2007). Using electro-antennography, Vereecken et al. (2007) identified physiologically active compounds in solvent extracts of virgin females. Electro-antennography detects changes in electric potential from an insect antenna exposed to an organic compound stimulation that are due to the superposition of simultaneous membrane depolarisations of numerous receptor cells, which are indicative of an output from the antenna to the brain (Schiestl \& Marion-Poll 2002). The existence of this output means that the organic compound stimulates receptor cells, and has thus a physiological effect in the brain. Vereecken et al. (2007) detected an individual variability in the physiologically active compounds that is higher among than within populations of Colletes cunicularius, which showed the existence of population-specific dialects within a multidimensional "olfactory landscape". The comparisons of the preferences of male from known provenance for synthetic copies of female sex pheromones showed that they were attracted significantly more often by odor types from allopatric populations, which is an excellent way to prevent mating with kin and inbreeding.

\section{The romance}

Each of the three mechanisms mentioned above that Hymenopterans developed to avoid inbreeding or to loose time and energy with previously courted partners (i.e. kin and mate recognition, protandry and dispersal) plays a role in the co-evolutionary relationships between Ophrys species and their pollinators, and hence has a potential role in speciation and adaptive radiation. Ophrys flowers simulate and manipulate insect kin and mate recognition mechanisms to attract their pollinators. Ophrys flowers are available when adult insect males emerge from their pupae, before the availability of insect virgin females. Pollinated Ophrys flowers emit anti-aphrodisiac compounds that are reluctant for pollinator males, similar to those pheromones that are produced by mated Hymenopteran females to avoid further courtships, as documented in the couple Ophrys sphegodes Andrena nigroaenea (Schiestl \& Ayasse 2001). We will review the empirical evidences associated to each of these three mechanisms, and where appropriate we will develop particular topics that will help to understand how intra-specific competition between plants will drive the different speciation mechanisms that entail the adaptive radiation in Ophrys.

\subsection{Pollinator attraction by manipulation of kin and mate recognition mechanisms \\ 2.1.1 Pseudo-pheromones mimic attractive insect female sexual pheromones}

The potential role of organic compounds emitted by Ophrys flowers in the attraction of pollinators was brought up to date by Kullenberg (1961) ca. $40 \mathrm{yr}$ after the pioneering work of Kullenberg, who was the first to suggest the attraction of male pollinators by flower odor (Correvon \& Pouyanne 1916a, b, Pouyanne 1917, Correvon \& Pouyanne 1923, see below). The hypothesis of Kullenberg (1961) was worked out by Berg-Karlson and her team (reviewed in Borg-Karlson et al. 1990). She was the first to use electrophysiological tests (gas chromatography coupled with electro-antennographic detection, GC-EAD and with mass spectrometry, GC-MS) to identify organic compounds emitted 
either by Ophrys flowers or by extracts of their female pollinators that had the potential to attract male pollinators. Using a sample of 30 Ophrys species, she identified compounds that triggered pollinator attraction but not those that were the releasing factors of copulation behavior. These results were confirmed by behavioral tests in which experimenters assessed the attraction of free flying pollinators by candidate organic compounds spread on insect dummies. This led her to propose the hypothesis that Ophrys flowers produce only 'second-class attractive compounds' and are neglected once the pollinator females are present.

However, further experiments demonstrated that organic compounds emitted by Ophrys flowers had the property to initiate pollinator copulation behavior (Schiestl et al. 1999, 2000). The similarity between the organic compounds emitted by Ophrys sphegodes flowers and the sexual pheromones present in cuticular extracts from females of their pollinators (the males of the solitary bee Andrena nigroeanea) was here again assessed by GC-EAD and GC-MS. Results indicated a huge similarity of biological activity between female sexual pheromones and of Ophrys flower odor bouquet, which translated into comparable composition and relative proportion of biologically active organic compounds. Synthetic, analogous blends of these compounds (C21 to C29 n-alkanes and nalkenes) applied to female dummies triggered copulation attempts by male pollinators (Schiestl et al. 1999, 2000). In all Ophrys species investigated so far, such copulation attempts of pollinators can only be elicited by a blend of organic compounds that is similar in composition and quantity to the female sexual pheromones of the pollinator (Schiestl et al. 1999, Ayasse et al. 2000, Schiestl et al. 2000, Schiestl \& Ayasse 2002, Ayasse et al. 2003, Mant et al. 2005, Ayasse 2006, Stökl et al. 2005, 2007, 2008, 2009, Gögler et al. 2009, 2011, Vereecken \& Schiestl 2008, Cuervo et al. 2017, Gervasi et al. 2017).

The composition of the blend of organic compounds forming sex pheromones and their floral analogues seems to vary according to the systematic position of their Hymenopteran pollinators (Cuervo et al. 2017). Wasp-pollinated Ophrys species would attract their pollinators by using polar hydroxyacids, Eucera-pollinated Ophrys species would use a mixture of polar and non-polar compounds and Andrena-pollinated Ophrys species would use non-polar hydrocarbons (Cuervo et al. 2017). This variation in attracting organic compounds emitted by the flowers would correspond to the above mentioned several pollinator shifts during the evolutionary history of the Ophrys genus (Breitkopf et al. 2015). Those shifts would have promoted the rapid diversification observed within several clades (Figure 1, Breitkopf et al. 2015). However, this latter assumption is not yet convincingly demonstrated. Firstly, there is no one-to-one relationship between the clades in which rapid diversification occurred and the attraction of a single pollinator family, i.e. Andrenidae (Table 1) (Gaskett, 2011; Breitkopf et al., 2013; Joffard et al., 2018). Secondly, the analyses of the bouquet composition of pheromone analogs within the Ophrys insectifera clade, which is the most basal clade according to almost all phylogenies using DNA sequence data, showed the simultaneous emission of both esters and non-polar hydrocarbons (Joffard et al. 2016, Gervasi et al. 2017). Moreover, among the three species in this clade, $O$. aymoninii, which is the only one to be pollinated by a species of Andrena (A. combinata), is also the only one to emit esters (Joffard et al. 2016). Unfortunately, we still lack data on the attractiveness of these compounds on the wasps that pollinate $O$. insectifera.

Whatever the evolution of composition of the floral analogues of sexual pheromones, the main message we draw from these studies is the striking similarities in their composition and relative amounts in orchid labella and attractive female bees. These similarities support the view that a 
specific blend of organic compounds is the crucial key innovations of Ophrys for pollinator attraction from a distance, and, importantly, for the initiation of pollinator male copulation behavior.

Table 1. Proportion of Ophrys species forming the two rapidly diverging clades identified by Breitkopf et al. (2015) that are attracted by different families of pollinators. The number of species in each clade is in brackets. Ophrys systematics following Delforge (2016); pollinator data from Gaskett (2011), Delforge (2016) and Paulus (2018).

\begin{tabular}{|l|c|c|c|c|c|c|}
\hline & \multicolumn{5}{|c|}{ Pollinators } \\
\hline Clade & Andrenidae & Apidae & Colletidae & Megachilidae & Scarabeidae & Sphecidae \\
\hline O. fusca $(\mathrm{n}=72)$ & 0.85 & 0.08 & 0.03 & 0.01 & 0.03 & 0 \\
\hline O. sphegodes (n=72) & 0.40 & 0.26 & 0.08 & 0.24 & 0 & 0.01 \\
\hline
\end{tabular}

\subsubsection{Ophrys intraspecific variation in organic compounds}

In Ophrys sphegodes, Ayasse et al. (2000) identified 106 compounds in the odor bouquets of flower extracts. GC-EAD revealed that only 24 of them had a physiological activity on pollinator males by triggering stimulation of their antennae in GC-EAD tests. Their data indicated unambiguously variation among inflorescences of the same species in GC-EAD active compounds. Moreover, flowers of the same inflorescence had a higher similarity in odor bouquet than flowers of different inflorescences, but differ in their relative proportion of GC-EAD active aldehydes and esters. The authors proposed that this difference is important to avoid pollinator habituation, which favors the successive visit of flowers of the same inflorescence by a given pollinator. Accordingly, during behavioral experiments, $2 / 3$ of the males who visit one flower visit a second flower on the same inflorescence. Mixtures of GC-EAD active compounds from inflorescences separated by $30 \mathrm{~km}$ were significantly more different among populations than within populations, which suggests variation of olfactory signals among metapopulations. Here these differences were due to variation in the relative proportions of $n$-alkanes and $n$-alkenes within the blend of active GC-EAD organic compounds. Finally, GC-EAD physiologically active compounds showed less intra-specific variation in the odor bouquets as compared to non-active compounds, which suggests that the variation of pollinator-attracting communication signals is constrained by some limits.

The investigation of chemical compounds present in the flower labella of Ophrys exaltata was performed by Mant et al. (2005) using a split geographical design: individual variation was assessed among regions, among populations within regions and within populations. The authors identified 59 compounds, of which 22 were identified as behaviorally active in the pollinator Colletes cunicularius by inducing male landing and copulation attempts on compound impregnated dummies. Variance partitioning indicated that one third of the odor variation was due to individual differences in physiologically active compounds within conspecific populations. Moreover, most of the individual variation (60\%) was observed among region, i.e. among metapopulations, whereas a small fraction of the variance (7\%) was observed among local populations. Strikingly, as in the previous study of Ayasse et al. (2000), inactive compounds present in the flower labella were much more different amongst individuals: variance partitioning indicated $95 \%$ of within population differences for behaviorally inactive compounds. 
Vereecken and Schiestl (2008) sampled plants and pollinators in 15 populations (13 allopatric and 2 sympatric) of the same plant-pollinator couple Ophrys exaltata - Colletes cunicularius. They focused on odor bouquets made by 3 key organic compounds that were detected by male bees. They showed that odor compounds triggering stimulation of the male bee antennae, the so-called physiologically active compounds, differ markedly between orchid flowers and female bees, irrespective of their geographic origin. This difference in odor bouquets was even consistent in the two sympatric populations. Besides, behavioral tests indicated that pollinators showed a marked preference for the odors of females from allopatric populations, i.e. among metapopulations, which confirms the previous results of Vereecken et al. (2007). Finally, males were always more attracted in behavioral tests by Ophrys flowers than by female bees. Given than the absolute amount of organic compounds produced by flowers and female bees is similar, it is the change of the relative amount of the three organics compounds used in this experiment that was responsible for the higher attractiveness of the orchid blends. This result was confirmed by manipulating the natural ratios of the three key compounds in natural sex pheromone extracts of local females of Colletes cunicularius. By adding synthetic hydrocarbons to change the female bees' natural proportions into the mean ratios found in orchid floral odor samples, there was a significantly higher behavioral activity in male bees that was thus caused by these deviant ratios in the three key compounds of the female sex pheromones.

Altogether, we can conclude from these experiments that within a given Ophrys species, each individual plant has its own olfactory signature from a pollinator viewpoint, which is produced by varying the relative amounts of physiologically active organic compounds produced by the flowers. Moreover, in Ophrys sphegodes, even if flowers of the same plant have a more similar olfactory signature than flowers of different plants, they differ from each other by the relative amount of some organic compounds. Finally, these three experiments indicate significant differences in the odor signatures of individuals belonging to different metapopulations. Two research axes require further interests: (1) the generality of this similarity between flowers of the same plant relatively to flowers from other plants, which was demonstrated on only one species and (2) the potential roles of the GC-EAD inactive organic compounds that seem more variable than the active organic compounds. We suggest that two non-exclusive explanations might generate the latter pattern, i.e. (1) a pollinator-mediated selection to maintain the relative ratios of a physiologically active organic compounds (Raguso 2008), and (2) a bet hedging strategy (e.g. Beaumont et al. 2009) inducing a high variability of physiologically inactive compounds to produce new molecules that are potential pollinator attractors.

\subsubsection{Intraspecific competition drives random crossing of peaks in the olfactory landscapes}

Highly species-specific mutualistic or antagonistic interactions between plants and pollinators have been suggested to be mediated by a few, system-specific compounds through 'private channels' (Raguso 2008; Chen et al 2009). However, contrarily to the highly species-specific mutualistic interactions like in the fig - fig wasp system in which mutual benefits depend on the consistency of plant olfactory signals over time (Chen et al. 2009), the olfactory signaling in the Ophrys - pollinator system cannot be stabilized over time due to the constraints imposed by the mnemonic pollinators, which counter-select the evolution of private channels (Dormont et al. 2019).

Hitherto, two different processes are invoked to explain the high level of within-species interindividual heterogeneity in organic compounds emitted by Ophrys flowers. On one hand, Vereecken 
and Schiestl (2008) proposed a proximal explanation based on pollinator male preferences for novel signals. They suggested that pollinator populations are "probably subjected to inbreeding", which makes such preferences for novel signals adaptive because it promotes outbreeding, i.e. it avoids sibling mating. They mentioned that such preferences for novel signals are regarded as a common emerging feature in animal cognitive processes, and that studies have demonstrated that this phenomenon can be an important driving force behind signal evolution (e.g. Lynn et al. 2005, Cate \& Rowe 2007, Dormont et al. 2019). On the other hand, Schiestl (2005) proposed an ultimate explanation based on negative frequency-dependent selection. He mentioned that deceptive orchids often show high variability in floral signals, which may be maintained by negative frequencydependent selection, since pollinators can learn and subsequently avoid common deceptive morphs more quickly than rare ones. In the same vein, Ayasse et al. (2010) considered that the evolution of floral variation in Ophrys is an extreme form of negative frequency-dependent selection in which two similar individuals are counter-selected.

We emphasize here that the first, proximal explanation is consistent with the current knowledge of pollinator biology presented above, i.e. the high inbreeding risk associated to the haplodiploïdy mechanism of sex determination in Hymenopterans, and the evolution of individual chemical signature to favor kin and receptive mate recognitions (Barrow et al. 1975, Wcislo 1992, Ayasse et al. 2001, Smith \& Ayasse 1987). We found less support for the second, ultimate explanation. In negative frequency-dependent selection the fitness of discrete phenotypes increases as its frequency in the population decreases, leading to maintaining balanced polymorphism, i.e. the long-term coexistence of several discrete phenotypes (i.e. morphs) that are selected successively according to their frequencies within populations (Brisson 2018 and references therein). Such negative frequency-dependent selection is key in polymorphic plants using the food deceptive pollination syndrome. In these species the preference of pollinators for the rarer phenotype progressively increases the frequency of this morph over generations, which progressively decreases its attraction for the benefit of the formerly frequent phenotype that became rarer and rarer and hence more attractive (e.g. Gigord et al. 2001, but see Jersáková et al. 2006). The high interindividual differences in Ophrys flower odor profiles do not correspond to such a coexistence of discrete phenotypes. Accordingly, we suggest that the variability of the biologically active and inactive organic compounds emitted by Ophrys flowers is the result of an intra-specific competition for a limited resource, i.e. their mnemonic pollinators. Such intense competition for resource might promote the individual variation in flower phenotypes of organic compound emission, and this increased diversity might arise with plasticity alone, without the genetic changes commonly assumed by theory (e.g. Svanbäck \& Bolnick 2007). Whatever the relative role of genetic or epigenetic mechanisms, we suggest that the end result of this high individual variability in organic compounds emission is the random crossing of peaks in the olfactory landscapes (Svensson et al. 2014) surrounding mate searching male insects randomly in its foraging area, and the emission by chance of a particular blend of odors attracting a new species of pollinator. Finally, we predict that if the high competition for mnemonic pollinators generates random inter-individual variability in organic compound, there should be a skewed reproductive success in Ophrys populations, some individual plants matching the odor preferences of their pollinators better than others. Some indirect evidences document that it is indeed the case, like the huge accumulation of pollinia within some flowers (Figure 2). 


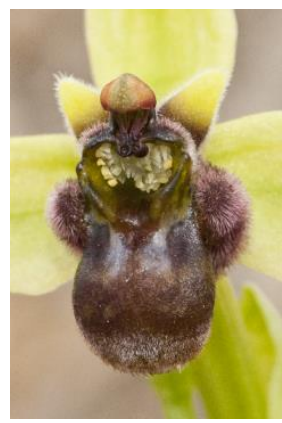

Figure 2. Flower of Ophrys bombyliflora with its stigmatic cavity filled by pollinia. Picture by courtesy of Jean Claessens.

\subsubsection{Evidences of intra-specific competition and pollinator limitation}

There are three indirect evidences of pollinator limitation in Ophrys that might induce intra-specific competition among Ophrys conspecific individuals. Firstly, the three studies we are aware that analyze Ophrys individual fitness in a spatially-explicit context showed a negative relationship between reproductive success and conspecific density. This general pattern is in good agreement with a higher competition for pollinators in denser plant populations, and hence advocates for pollinator limitation. Vandewoestijne et al. (2009) investigated during 2 successive years the reproductive success of all plant individuals in 4 populations of Ophrys sphegodes, 4 populations of Ophrys fuciflora and 5 populations of Ophrys insectifera (one of them with 3 sub-populations). Female individual reproductive success was approximated as fruit set (ratio of pollinated flowers or inflated capsules divided by the total number of flowers in a given inflorescence). For all three species examined, the female individual reproductive success increased with increasing nearest neighbor distance (meaning the shortest distance to the nearest flowering individual). Moreover, female individual reproductive success generally increased with decreasing population density (number of individual plant $/ \mathrm{m}^{2}$ ) and increasing habitat patch elongation (width divided by length). The latter parameter was included because a potentially larger number of pollinators are likely to encounter patch boundaries (and consequently immigrate into or emigrate out of the patch) when the perimeter increases. Fruit set success was also variable between years but in a similar way among populations and across species. Gervasi et al. (2017) investigated the female individual reproductive success of 300 plants of the phylogenetically closely related Ophrys insectifera and Ophrys aymoninii that were randomly selected within 6 populations where the two species lived in sympatry. They showed that in both species the pollination success of a given plant was negatively related to the number of conspecific individuals within a $2 \mathrm{~m}$ radius. Borràs \& Cursach (2018) compared the reproductive success among 7 populations of Ophrys balearica during two successive years. They found that fruit set was higher in $2 \times 2 \mathrm{~m}$ quadrats where density was lower (1-10 individuals) compared to quadrats with higher density. Moreover, those flowers that were located at the periphery of the populations had a higher male reproductive success (measured by pollinia removal) than those that were at the center of the populations.

Secondly, many publications report that Ophrys flowers have a low to a very low female individual reproductive success (e.g. Neiland \& Wilcock, 1998, Claessens \& Kleynen 2011, 2016). This general pattern is in good agreement with the rarity of a pollination event for an Ophrys individual, and here again advocates for pollinator limitation. However, most of these publications do not mention the exhaustiveness of the sampling that is used to compute this basic component of fitness. As previously mentioned, Vandewoestijne et al. (2009) and Borràs \& Cursach (2018) showed that among the many parameters influencing the pollination of one or several flower(s) of a plant, both 
its position in space within the habitat relatively to habitat borders and its distance to its closer neighbor influence female individual reproductive success. More similar studies detailing exhaustively the within Ophrys population success in relation to those explicit spatially variables are clearly needed. Comparisons between male and female reproductive success estimated via the value of the Pollen Transfer Efficiency index is a promising criterion to evaluate the target of the intraspecific competition and its consistency across species and environmental contexts (Scopece et al., 2015).

Thirdly, the life history of Ophrys individuals is rather singular. They are long-lived species herbaceous species (up to $20 \mathrm{yr}$ : Wells \& Cox 1991, Hutchings 2010) that produce large number (5000-20.000/fruit: Arditti \& Ghani 2000, Paulus 2006, Claessens \& Kleynen, 2011, 2016, Sonkoly et al. 2016) of seeds of tiny size (300-700 $\mu \mathrm{m}$ length, 100-200 $\mu \mathrm{m}$ width: Galán Cela et al. 2014) and weight (11-20.10-7 g: Sonkoly et al. 2016). At the time of dispersal, Ophrys seeds consist of a spindleshaped, very slim seed coat that encloses an extremely small and simplified embryo formed of a spherical cluster of cells, which is a clear adaptation to wind dispersal (Arditti \& Ghani 2000). The embryo is unable to germinate on its own and has thus to engage in a mycorrhizal relationship with a fungus that sustains the development of the emerging seedling. Female individual reproductive success is thus dependent on the massive production of these wind-dispersed seeds that are able of long distance dispersal. Accordingly, Willems (1994) reported the finding of a single flowering individual of Ophrys apifera in a Dutch dune area that was 60-75 km apart from the existing populations of the species. Theory indeed predicts that selection for long distance dispersal of seeds mediates positive density-dependent pollinator limitation in plant populations using deceptive pollination (Ferdy et al. 1999).

An important research axis deserves further research interest. We are still lacking observational and experimental evidences of the link between the individual variation in odor bouquet and their reproductive success in a spatially explicit context, i.e. by taking into account Ophrys densities, habitat geometry and the distance to the closest neighbor within local populations, or the intensity of gene flow within metapopulations (see Sletvold et al. 2010, 2016). This research for a relationship between individual variation in odor bouquet and their reproductive success should also investigate the additive or interactive effects of potential explanatory variables influencing pollination success, i.e. pollinator abundances or weather conditions.

\subsubsection{Evolution of floral odor phenotypes and speciation}

Strong intra-specific competition due to pollinator limitation seems thus induce the high interindividual variability in organic compounds emitted by Ophrys flowers. We assume that flowers produce random blends of organic molecules to avoid habituation by their mnemonic pollinators. The consequences of this selective pressure for novelty in odor bouquets produced by the flower would be threefold: (1) the bouquet can be biologically inactive, (2) the bouquet matches the pheromone bouquet of virgin females of its usual pollinator species and (3) the bouquet can match the pheromone bouquet of virgin females of another pollinator species. In the first case, there would be no chance for reproduction, and the flower fitness is nil. In the second case, cross-pollination would be possible if a flower-pollinator encounter occurs in space and time, and the match between a particular flower odor bouquet and the sexual pheromones of its pollinator maintains the boundaries of Ophrys species by pre-zygotic isolation as previously mentioned. These two contrasted issues might explain the much higher variability of biologically inactive compounds relative to active 
ones mentioned previously. Even if the production of organic compounds is random, the nature and the proportion of biologically active compounds in the blend produced by the flower would be constrained by its evolutionary history, mainly by pollinator-mediated selection (Raguso 2008), and hence should be restricted to a narrower range of variation than biologically inactive compounds. In the third case, cross-pollination would be possible, and might end up in a speciation event, either by hybridization if the newly attracted pollinator is already pollinating another Ophrys species or a new species if the newly attracted pollinator is not involved in the pollination of another Ophrys species. Ayasse et al. (2010) discussed these scenarios in another framework (individual variation in odor bouquet is produced by chance and followed by negative-frequency dependent selection of plants by their pollinators).

\subsubsection{Speciation by hybridization}

We have supposed so far that one and only one pollinator species pollinated each Ophrys species. The comparative analysis of the pollinator networks among Euro-Mediterranean orchid species indeed indicated that Ophrys species are pollinated by a mean of 1.56 pollinator species, which is the lowest value for all orchid genera in this area (with a mean of 7.44 pollinator species across genera, and a maximum of 158 pollinator species for the food-rewarding orchid Neottia ovata) (Joffard et al. 2019). A close examination of the currently available data bases of Ophrys pollinators (Claessens \& Kleynen 2011, 2016, Gaskett 2011, Paulus 2018) indicate that Ophrys species with wide distribution ranges are reported to be pollinated by several, usually congeneric pollinator species. For instance, 6 species of Eucera bees (Apidae) were reported as pollinators of Ophrys bombyliflora (Paulus 2018), which has a wide circum-Mediterranean distribution. Such observations clearly require closer investigations especially if they are performed in different locations within the distribution area of the species. Indeed, subtle differences in flower morphology and phenological variation suggest that Ophrys bombyliflora is rather a constellation of species (Delforge 2005, 2016). Despite this bias towards higher number of pollinator species in widely distributed (complex of) species, the large number of hybrids (more than 600 currently described: Delforge 2016) even among the 9-11 different clades identified by molecular systematics based on DNA sequences (e.g. Breitkopf et al. 2015, Bateman 2018a) means that other species than its species-specific pollinator can visit a given Ophrys flower and eventually transfer pollinia.

Post-zygotic barriers seem rather rare in Ophrys (e.g. Scopece et al. 2007, but see Cortis et al. 2009, and Vereecken et al. 2010 discussed here below), and hybridization is considered as a source of evolutionary novelties that can ultimately lead to pollinator shifts and reproductive isolation (Cotrim et al. 2016). This scenario of pollinator shift is illustrated nicely by a case study by Vereecken et al. (2010). These authors analyzed the hybridization between two species of Ophrys (O. lupercalis and $O$. arachnitiformis) that belong to different clades according to Breitkopf et al. (2015) and that use contrasted pollination strategies. Males of Andrena nigroaenea (Hymenoptera, Andrenidae), pollinate $O$. lupercalis by introducing their abdomen into the stigmatic cavity of the flower and carry pollinia on their abdomen (abdominal position). Males of Colletes cunicularius (Hymenoptera, Colletidae) pollinate $O$. arachnitiformis by introducing their head in the stigmatic cavity of the flower and thus carry pollinia on their head (cephalic position). Experiments with the scent bouquet of their hybrids in Southern France showed low attraction for either pollinator of the parent species, but relatively greater attraction of a third species, Andrena vaga, which does not pollinate the parents. The scent bouquet of the hybrids includes odor compounds that are either absent from those of the parent species ( 2 organic compounds), or expressed only in very low concentrations. Such 
evolutionary novelty in hybrids suggests thus that hybridization may contribute to the high pollinator diversification in Ophrys. However, the existence of hybrids questions the generality of the one to one correspondence between a given Ophrys species and its species-specific pollinator. In their paper, Vereecken et al. (2010) published pictures of cross copulation attempts, i.e. C. cunicularius with $O$. lupercalis and A. nigroaenea with $O$. arachnitiformis. These pictures (Figure 3) showed that both pollinator species used both copulation positions, either cephalic or abdominal. In this particular case, F1 hybrids between $O$. lupercalis and $O$. arachnitiformis were not fertile, and back-cross pollination between $\mathrm{F} 1$ hybrids and their parent species did not result in seed production. Further analyses showed that $O$. arachnitiformis was diploid, $O$. lupercalis was tetraploïd and F1 hybrids were triploid, which might explain their sterility. Even if in this case of postzygotic isolation hybridization fails to produce a new species, this example illustrates the way speciation may occur through the production of a new bouquet of organic compounds by the Ophrys hybrid that attract a new pollinator species.

Another informative study is the analysis of Stökl et al. (2008) that investigated the pollinator syndromes in sympatric and allopatric populations of Ophrys lupercalis and O. eleonorae in Sardinia. $O$. lupercalis is widespread around the Mediteranean basin, whereas $O$. eleonorae is endemic to Sardinia and Corsica. These species are pollinated by Andrena nigroaenea and Andrena morio respectively, both using abdominal copulation position. The authors used an integrated approach that combined morphological and chemical analyses of flowers, and GC-EAD and behavioral tests on pollinators. Moreover, the authors realized genetic assignation of plants using AFLP markers, and compared Sardinian plants to specimens from Majorca where only $O$. lupercalis is present, and from Greece where only $O$. iricolor is present. $O$ iricolor to which $O$. eleonorae is vicariant, is thus a sister taxon widespread in the Eastern Mediteraneas basin and pollinated by Andrena morio only.

Morphological investigations of flowers indicated in some Sardinian population the presence of phenotypes that had shapes and colors of the labellum intermediary between the two parent species. Those plants were considered as hybrids between $O$. lupercalis and $O$. eleonorae. As in other cases of flowers pollinated by Andrena species (Schiestl \& Ayasse 2002, Stökl et al. 2005, Stökl et al. 2009), there was a large overlap in the EAD hydrocarbons released by the flowers of the two Ophrys species. Here, all the organic hydrocarbons were present in the bouquet of the two parent flowers and of their hybrids, albeit in significantly different proportions in 17 cases out of 24 for the parents. The proportion of organic hydrocarbons that were present in the bouquet of hybrids significantly differed from both parents in only 1 case out of 24 . Behavioral experiments revealed that ca. $20 \%$ of the flowers from both parent species were attractive to both pollinators, and that this proportion was higher in hybrids (Table 2). 

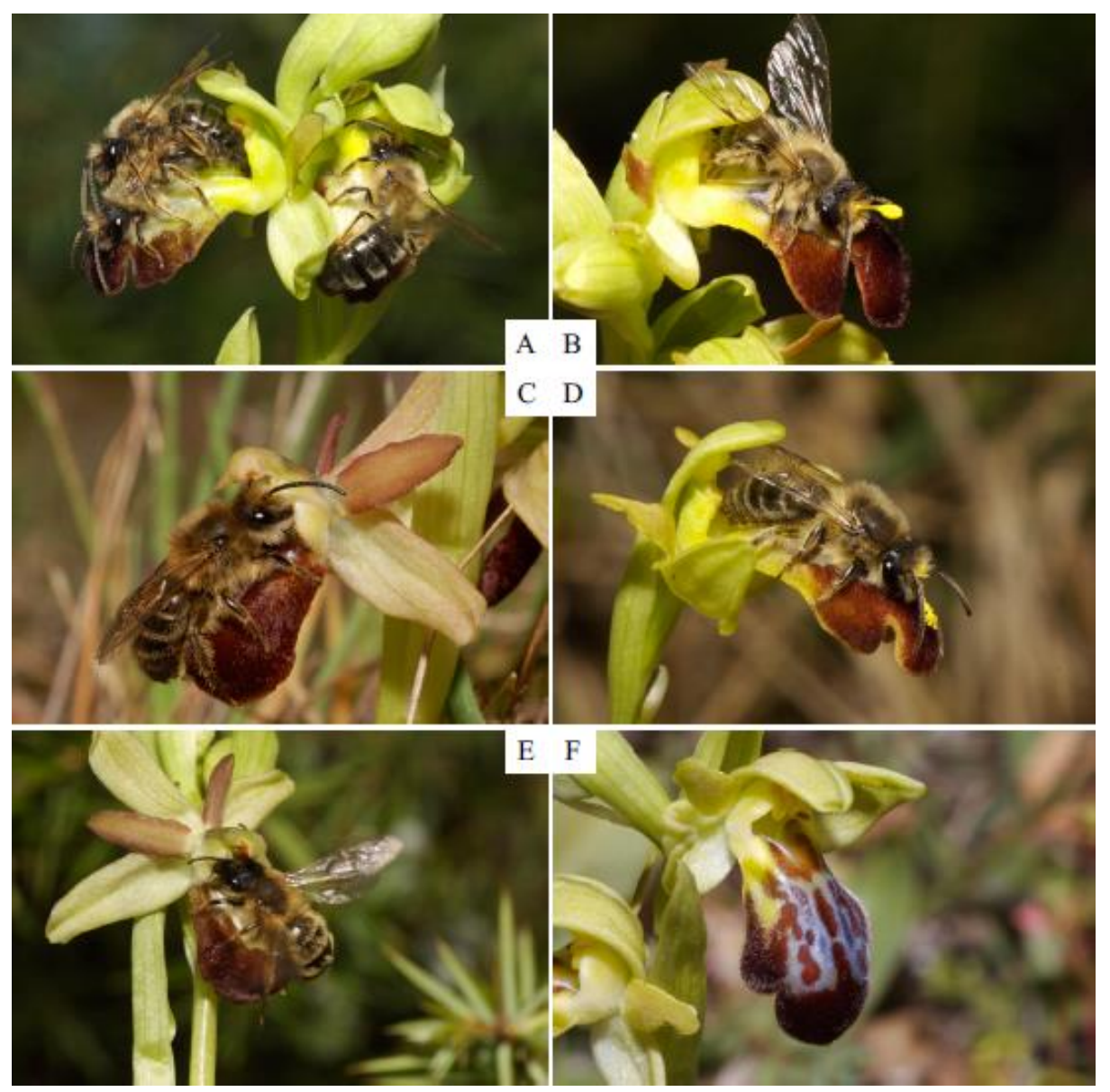

Figure 3. A. Copulation attempts of males of Andrena nigroaenea (Kirby) (Hymenoptera, Andrenidae) in both the "abdominal" and the "cephalic" positions on the flower labella of Ophrys lupercalis; B. Copulation attempt of male of $A$. nigroaenea in the "abdominal" position on a flower of $O$. lupercalis with pollinaria on its head; $C$. Copulation attempt of male of Colletes cunicularius (L.) (Hymenoptera, Colletidae) in the "cephalic" position on the flower labellum of $O$. arachnitiformis; D. Copulation attempts of male of $C$. cunicularius on the flower labellum of $O$. lupercalis with pollinaria on its head; E. Pseudocopulating male of $A$. nigroaenea in "cephalic" position on the flower labellum of $O$. arachnitiformis; $\mathrm{F}$. Detail of a flower of the natural hybrid between $O$. arachnitiformis and O. Iupercalis. All photographs by N.J. Vereecken. Reproduced from Vereecken et al. (2010), Open Access.

Genetic assignations revealed that $O$. lupercalis individuals from Majorca clustered together and grouped with most $O$. lupercalis individuals from Sardinia and with some hybrids. O. eleonorae

Table 2. Proportion of potted flowers that released copulation attempts by males of the corresponding pollinators in the field. Number of tested flowers between brackets (data from Stökl et al. 2008).

\begin{tabular}{|l|c|c|c|}
\hline Species & $\begin{array}{l}\text { Andrena nigroaenea } \\
\text { alone }\end{array}$ & Andrena morio alone & $\begin{array}{l}\text { Both Andrena } \\
\text { species }\end{array}$ \\
\hline Ophrys lupercalis $(\mathrm{n}=10)$ & 0.80 & 0 & 0.20 \\
\hline Ophrys eleonorae $(\mathrm{n}=9)$ & 0.22 & 0.56 & 0.22 \\
\hline Hybrids ( $\mathrm{n}=14)$ & 0.36 & 0.36 & 0.28 \\
\hline
\end{tabular}


individuals from Sardinia and O. iricolor individuals from Greece grouped in two different clusters ${ }^{3} . O$. eleonorae individuals from Sardinia clustered with many hybrids and with a couple of Sardinian $O$. lupercalis individuals. Some hybrids were intermediary between Sardinian O. eleonorae and the cluster of $O$. iricolor individuals from Greece. Altogether, these results suggest that the cross attraction of nonlegitimate pollinators induced introgression between the genomes of the two parent species in Sardinia. This introgression is much higher in $O$. eleonorae individuals that were genetically indistinguishable from most hybrid individuals. The swamp formed by Sardinian $O$. eleonorae individuals and most hybrids indicates how a parent species can be absorbed by a hybridogenetic taxon and eventually can form a new species. This example demonstrates also clearly the determinant role of pollinator attraction by the plant in the maintenance or in the breakdown of reproductive isolation between Ophrys species.

In our quest of the drivers of adaptive radiation in Ophrys, this example illustrates that speciation by hybridization might appear as a misleading explanation of the rapid increase in species number. Indeed, one of the parent species is progressively absorbed by the hybrid, leading to the net result of two species, one new and one parent, instead of the two parent species. However, when this process of speciation of hybridization concerns metapopulation lineages that are located in restricted parts of the distribution ranges of the two parents, the absorbed parent species can maintain pure metapopulation lineages in other parts of its ranges. Altogether, the net result of the speciation by hybridization process could thus contribute to an increase in the species number.

\subsubsection{2 de novo speciation}

Here we argue that the high inter-individual variation in odor blends induced by strong intra-specific competition for limited pollinators induces the production of new (blend of) organic compounds that attract males of insects that were not used as pollinators by other Ophrys species. We will illustrate such de novo speciation events by two well documented examples.

The couple formed by Ophrys speculum and its pollinator the wasp Dasyscolia ciliata (Hymenoptera, Scoliidae) is famous in the relatively short history of investigations of the Ophrys pollinator syndrome. It is indeed on this couple of species that the French naturalist Maurice Alexandre Pouyanne (Correvon \& Pouyanne 1916a, b, Pouyanne 1917, Correvon \& Pouyanne 1923) described the species-specific attraction on, and the copulation attempts of male insects with, Ophrys flower labellum. He provided also experimental clues of the role of odor in this attraction. The blend of organic compounds that were responsible for the detection of the flower and the release of the male copulation behaviour was identified by Ayasse et al. (2003) using GC-EAD and behavioral experiments. Male antennae reacted to 10 components that were identified as saturated

\footnotetext{
${ }^{3}$ These genetic differences confirm the specific differentiation between $O$. eleonorae and $O$. iricolor that are closely related species living in allopatry. The specific status of the Sardinian endemic O. eleonorae was already proposed by Devillers and Devillers-Terschuren (1994), but not followed by Stökl et al. (2008) in their initial publication that considers the Sardinian O. iricolor individuals as conspecific of Greek individuals. These authors mention the existence of $O$. eleonorae in a re-analysis of their data (Ayasse et al. 2010), but persist to consider $O$. iricolor only, despite the genetic differences they observed between individuals belonging to these two taxa. This is a nice example of the danger of unreliable taxonomic diagnosis: the direct consequence of the absorption of Sardinian $O$. iricolor by the hybrid $O$. iricolor $X O$. lupercalis would have been the apparition of a new species because $O$. iricolor persist in other part of its range. However, the direct consequence of the absorption of the Sardinian endemic $O$. eleonorae by the hybrid O. eleonorae $\mathrm{X} O$. lupercalis is the substitution of the endemic $O$. eleonorae by a new species.
} 
( $\omega$-1)-hydroxy and ( $\omega$-1)-oxo acids, aldehydes and ethyl esters. Three components of the blend (9oxodecanoic acid, 9-hydroxydecanoic acid and 7-hydroxyoctanoic acid) were identified for the first time in plants. The relative proportions of most of the GC-EAD-active compounds differed significantly between the orchid and its pollinator. However, the major component of the active compounds in the wasps (9-Hydroxydecanoic acid) was also a major component of the active organics in the orchid. Behavioral tests showed that Ophrys flowers were significantly more attractive to males than their own females; this was confirmed by the significantly larger attractiveness of female dummies impregnated by synthetic organic compounds corresponding to the blend of Ophrys flowers compared to real females. The authors suggest that the 3 -time larger quantity of 9-Hydroxydecanoic acid produced by individual flowers compared to female wasps is responsible for this difference, and suggest that the production of larger amount of male attractive scents is counter-selected in females by the risk of attracting predators and brood parasites by producing long-distance sex pheromones. This case study could be an example of the potential role of the many biologically inactive organic compounds that are produced by Ophrys flowers. We assume that individuals of an ancestral species produced by chance one or several of the compounds that attract males of Dasyscolia ciliata as a consequence of the rapid evolution of the metabolic pathways leading to the production of organic compounds, itself due to the strong selective pressure due to pollinator limitation. The production by Ophrys speculum of compounds that are unique in the vegetal kingdom is an indicator of the strength of this selective pressure.

The second example comes from the extensive investigation of the molecular mechanisms allowing reproductive isolation in the couple of sympatric and synchronic Ophrys sphegodes and $O$. archipelagi, two species that are phylogenetically closely related (e.g. Devillers \& DevillersTerschuren 1994). The first species is pollinated by Andrena nigroaenea (Hymenoptera, Andrenidae) and the latter by Colletes cunicularius (Hymenoptera, Colletidae). Despite their phylogenetic distances, males of these two solitary bees were attracted by the same family of organic compounds i.e. n-alkanes and n-alkenes (Mant et al. 2005). However, the major floral odor difference among the species was the proportion of different n-alkenes (Xu et al. 2011, Sedeek et al. 2014). A field experiment investigated the transfer of stained pollinia. Results indicated a perfect intra-specific match: whereas 15 and 21 flowers of Ophrys sphegodes and $O$. archipelagi received stained pollinia from insect pollinators, there was not a single interspecific transfer (Xu et al. 2011). Moreover, hand pollination showed that interspecific crosses induced fruit set in all the tested cases, and the viability of the seeds produced by these interspecific crosses was not significantly different from those issued from intraspecific crosses (Xu et al. 2011). Altogether, these results indicated the preeminence of prezygotic isolation mechanisms due to selective pollinator attraction in the maintenance of species boundaries within this couple of closely related Ophrys species (Xu et al. 2011). Careful investigations of the volatile alkenes produced by these species revealed the very functioning of this differential attraction of their pollinators. Ophrys sphegodes produces mostly alkenes 9 - and 12-alkenes (i.e. with double bounds in position 9 and 12, whereas $O$. archipelagi produced high levels of 7-alkene (i.e. with a single double bound in position 7). Two desaturase genes, SAD2 and SAD5 that encode for stearoyl-acyl carrier protein desaturases (SAD), are responsible for either 9- and 12-alkene or 7alkene production, respectively (Schlüter et al. 2011; Xu et al. 2012). SAD are soluble, nuclearencoded, and plastid-localized proteins that act on an acyl group and catalyze the introduction of a double bond into saturated fatty acid precursors of alkenes (Schlüter and Schiestl 2008, Schlüter et al. 2011); next, alkenes are produced via elongation of those unsaturated fatty acids (Xu \& Schlüter 
2015). The expression and enzymatic activity of SAD2 are typically high in $O$. sphegodes and low in $O$. archipelagi, whereas the expression of SAD5 is high in $O$. archipelagi and low in $O$. sphegodes. The molecular mechanisms and their genetic architecture involved in these differences are detailed in Schlüter et al. 2011, Xu et al. 2012, Xu \& Schlüter 2015). The pollinator of O. sphegodes (Andrena nigroaenea) appears to be attracted to 9 - and 12 -alkenes, whereas 7 -alkenes reduce this attraction (Xu et al. 2012). Conversely, the pollinator of Ophrys archipelagi (Colletes cunicularius) is attracted by 7-alkenes, whereas addition of 9- and 12-alkenes reduces this attraction (Xu et al. 2012). These pollinator preferences may have imposed divergent selection on odor bouquets between the two orchid species (Xu et al. 2012). Indeed, Sedeek et al. (2016) showed how the enzymatic activity of the ancestral proto-SAD5 protein generated both 7- and 9-alkenes; they proposed that the restriction of the current SAD5 to the production of 7-alkenes only, which is due to two amino-acid changes at a crucial position of the enzyme, results from pollinator-mediated selection. This latter example corresponds nicely to a scenario of random crossing of peaks in their surrounding olfactory landscape by some Ophrys sphegodes individuals over-expressing the proto-SAD5. The resulting production of both 7- and 9- alkenes could have reduced the attraction of Andrena nigroaenea and initiated the attraction of Colletes cunicularius. Those mutants that possess the two amino-acid changes at a crucial position, and consequently that produce only 7-alkenes, would have realized the final step of de novo speciation by insuring the integrity of the attraction of a new legitimate, species-specific pollinator. Contrarily to the previous case study, here speciation occurred through changes in biologically active compounds.

Such de novo speciation events by pollinator shift can only occur if several individual plants cross randomly at the same time the same peak of the olfactory landscapes within the home range of the newly attracted pollinator, which might seem at first sight a rather stringent condition. However, the lifestyle of Ophrys is particularly suited to fulfill this requirement because it favors gene flow between newly speciated Ophrys individuals in time and space. As previously mentioned, Ophrys individuals are long-lived (up to $20 \mathrm{yr}$ ), which means that flowers with a particular blend of odors are presented repeatedly within a population over a long time period, provided that flowers of a given plant produce the same blend of odors over its reproductive life. Such data are not yet available. Moreover, as previously suggested, the strong competition for mnemonic pollinators should increase the production of new blends of organic compounds, both by the generation of new molecules and by the change of the relative concentration of existing compounds within the blend. Accordingly, the long-term availability of new blends of organic compounds within a population should increase the chance that two or more Ophrys individuals would be cross-pollinated by a new pollinator species. Besides, males of solitary bees may forage over distances of hundred meters to kilometers, which increase their probability of encounter with individual plants producing similar new odor bouquets. However, even if the lifestyle of Ophrys seems particularly suited for de novo speciation, the higher estimated diversification rate (between 4 and 8 lineages million $\mathrm{yr}^{-1}$ : Breitkopf et al. 2015) indicates that speciation remains a relatively scarce event.

\subsubsection{Directional selection of flower shape, colors and hairiness}

Once a new Ophrys species is produced, either by hybridization or by de novo speciation, we anticipate both inter- and intra-specific competition. Inter-specific competition with parent plants of the new taxon might take place if its new blend of attractive organics is not strictly different from the odor bouquets of the parent(s). Also, with the increase of its population size, intra-specific competition between individual plants of the new taxon for the new pollinator will gradually occur. 
Besides the production of an original blend of organic compounds, another way for Ophrys flowers to solve these competition issues, and thus to strengthen their attraction to their legitimate pollinator is to increase their morphological resemblance with his female. We expect thus a directional selection resulting in an ever more perfect floral imitation of the corresponding insect females. The end point of this directional selection would be a perfect match to the pollinator eyes between the flower and the female body. The gradual evolution towards a perfect match is even evident for human observers (Figure 4).

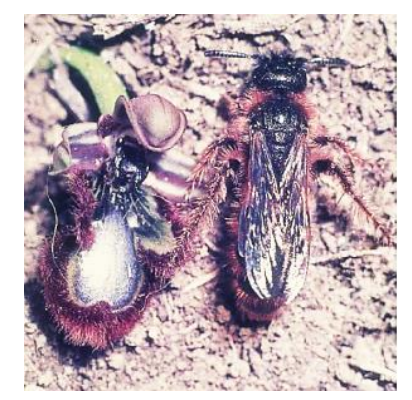

Figure 4. Match between an Ophrys speculum flower and the female of her pollinator. The glossy blue speculum on the labellum of the flower corresponds to the bluish wings of the pollinator's female, the lateral lobes of the labellum mimic her legs and the brown hairs bordering the labellum are similar to her pilosity. Picture reprinted from Paulus 2006, with permission.

\subsubsection{The role of flower color, shape and pubescence in pollinator attraction}

As previously mentioned, pollinators are attracted by analogs of sexual pheromones from a distance of ca. 5-10 m (Paulus 2006). Then, visual signals take over at close distance to trigger the landing of the pollinator on the labellum of the flower. Ophrys flowers hijack two categories of visual signals used by pollinators to detect their females, i.e. the color of the flowers visited females looking for food, and the color, shape and pubescence of the body of the female. These visual signals are produced by the upper part (perianth) and the lower part of the flower, respectively.

The importance of signals of the first category has been investigated using Ophrys heldreichii as model species in a suite of experiments (Spaethe et al. 2007, Streinzer et al. 2009). Results showed a significant increase in pollinator attraction through an additive effect of visual signals (a large pinkish perianth) at close distance $(<60 \mathrm{~cm}$ ) to the olfactory cues produced by the labellum. Spaethe et al. (2007) proposed that selection may have favored the spectral resemblance between the pinkish perianth of the flowers of $O$. heldreichii and the overall reflectance of the food plants visited by foraging females of the pollinator, Eucera berlandi. Moreover, Streinzer et al. (2009) showed that the color of the perianth is not the only visual cue used to locate flowers: bees also use an achromatic visual channel that relies on information from green-sensitive photoreceptors for detection of flowers at closer range $(<30 \mathrm{~cm})$. In attempt to generalize these results, Spaethe et al. (2010) found that whatever their evolutionary relatedness, the perianth of Ophrys flowers pollinated by Andrena and Eucerini bees have significantly different colors, i.e. green for Andrena pollinated species and pink for Eucerini pollinated species. They interpret this finding as the consequences of differences in the mate-locating strategy used by these two groups of bees, which is more visualbased in Eucerini bees. They suggest that the acquisition by Eucerini bees of a colored perianth matching the reflectance of the surrounding flowering plants might be at the cost of potential pollen loss due to the attraction of unspecific visitors. Even if the existence of this cost has to be formally demonstrated, this significant genus-wide dimorphism in perianth colors according to the pollinator 
sensory equipment and mate-locating strategy fits nicely the hypothesis of a directional selection leading to the design of the most efficient pollinator attracting signals.

A key question here is the co-variation between visual and olfactory signals: is there a relationship between a polymorphism in flower color and variation in analogs of sexual pheromones? This co-variation should curb the evolution of each signal, and would invalidate our scenario of random crossing of peaks in the olfactory landscapes followed by directional selection on flower phenotypes. The answer to this question is provided by the study of Vereecken \& Schiestl (2009). Two morphs of individuals of Ophrys arachnitiformis are differentiated by the color of their perianths, which are either green or white. The frequency of each morph varies among populations, ranging from $100 \%$ of green morphs along the Rhône to almost $100 \%$ of white morphs in south-east France and northeast Spain (Vereecken \& Schiestl 2009). The monitoring of individuals showed that color morphs are stable in space and time, which suggests that the polymorphism observed has a genetic basis (Vereecken \& Schiestl 2009). These authors worked in two populations where both morphs cooccur in southern France, which were located ca. $20 \mathrm{~km}$ apart. They analyzed the organic compounds that attracted the pollinator of Ophrys arachnitiformis, i.e. males of Colletes cunicularius, to look for differences in blend composition between the morphs. The relative and absolute amounts of active compounds were identical in the two morphs. Moreover, behavioral tests in the field using dummies showed that the olfactory signal was the only driver of pollinator attraction. Neither the presence of a perianth nor its color influenced visitation rates of scented dummies by patrolling males of $C$. cunicularius. Variation in visual signals of the first category seem thus decoupled from olfactory signals at least in this case study. However, this example remains punctual and focused on variations of perianth color only; this is why we encourage new investigations on the effects of variations of labellum color and olfactory signals on pollinator attraction aiming at generalizing this case study. Recent reviews about intraspecific variations of floral odor and on color-odor associations in the orchid family as a whole highlighted the existence of a broad variety of situations that depend on the biochemical constraints and the evolutionary context linked to the pollination strategy and the habitat requirement of the species (Delle-Vedove et al., 2017; Dormont et al., 2019)

In the second category of visual signals, the shape, color and pubescence of the lower parts of the Ophrys flower may correspond to visual signal of the body of the female of their pollinator. Rakosy et al. (2017) investigated how the shape of the labellum is used by the flower to attract and manipulate her pollinator. By using 3-D techniques, these authors showed how several crucial points of the labellum of Ophrys leochroma brought their pollinators (males of Eucera kullenbergi) in position for an efficient pollination. This study revealed that the labellum shape has an essential and so far underestimated role in ensuring effective pollination by mechanically guiding pollinators towards the reproductive structures of the flower, and by offering them gripping points on the labellum similar to those on the female body. Moreover, Rakosy et al. (2017) showed that pollinators were significantly less effective in interacting with experimentally manipulated flowers with a shape that was altered to resemble to those Ophrys species pollinated by other Hymenopteran genera. These authors proposed the existence of mechanically-active and -inactive components on the labellum morphology, which appears to be under different pollinator-mediated selection pressures similarly to biologically-active and -inactive compounds present in the olfactory signals. Accordingly, mechanically active components of the flower form could reflect adaptations to the interaction with particular pollinator groups, while inactive components can vary more freely. Such results are 
promising and valuable insights into the mechanisms driving the morphological diversification of the functionally different components of Ophrys flowers.

A classification of the shape, color and pubescence of the lower parts of the Ophrys flower was proposed by Paulus (2006), who distinguished three classes of Ophrys flowers according to the intensity of their match with the body of the female of their pollinator. In the first class, there is a perfect match between the shape and the color of the flower and its pollinator. This is the case of Ophrys speculum, where "the blue mirror of the labellum imitates the blue iridescence of the female's wings and the reddish-brown hair-like structures on the labellum imitate the red body hairs of the female wasp" (Paulus 2006). Also, "the reddish brown colour of the hairs of the labellum matches almost miraculously the color of the body pubescence of the female wasp" (Paulus 2006). This color is typical of the female of the wasp Dasyscolia ciliata ciliata ${ }^{4}$, whose males are used as pollinator by Ophrys speculum in Western Mediterranean. In the Eastern part of its range, Ophrys eos, the vicariant of $O$. speculum is pollinated by the vicariant wasp Dasyscolia ciliata araratensis, in which females have a dark brown body pubescence. Accordingly, the pubescence of the margins of the labellum of the flowers of the vicariant Ophrys eos is conspicuously darker (Paulus 2006). In the second class, the similarity between the flower and the body of the female of its pollinator is less obvious. Among the examples proposed by Paulus (2006), we select the flowers of Ophrys kotschyi and $O$. cretica that are both pollinated by the bee Melecta tuberculata (Hymenoptera, Melectidae). These two species belong to two different lineages, i.e. the clade of $O$. umbilicata (O. kotschyi) and the clade of $O$. holoserica (O. cretica) (Figure 1 ), as demonstrated by the analysis of supposedly neutral (nrlTS and a choroplast intergenic spacer region: Rrn5-Trn) DNA markers (Sramko et al. 2011). Despite their different origin, flowers of both species show marked convergence in colors: their green perianth is tinged with pink, the bottom of their stigmatic cavity is white (which is very rare in Ophrys flowers), the white, complex $\mathrm{H}$-shaped macula of the labellum is large and shows a strong contrast with the dark brown background color of the labellum, like the large white margins of the upper part of the labellum. These color patterns are obviously imitations of the repartition of black and white patches on the body of females of Melecta tuberculata. Ophrys flowers of the third class imitate only the background coloration of the females of their pollinator. Most representatives of this category belong to two recent clades, i.e. the clade of Ophrys fusca and the clade of $O$. sphegodes (Paulus 2006). In this latter case, we compare the labellum of $O$. sphegodes, which matches the reddish brown or gray brown color of the female of Andrena nigroaenea, whereas the deep dark blackish brown of $O$. incubacea matches the dark body colour of Andrena morio.

These three classes of flowers were considered as indicative of the role of the visual signaling in the mating behavior of their respective pollinators by Paulus (2006), from very important (category one) to not important (category three). Here, we suggest an alternative, more parsimonious explanation that is rooted in the evolutionary history of the different clades of Ophrys. We propose that these three classes correspond to a gradient in the evolution towards an ever more perfect match between the Ophrys flowers and the visual signals associated to the recognition of their mate by Ophrys pollinators. This evolution is fueled at each generation by a directional selection based on the higher reproductive success of the flowers that display the best visual signals. Our suggestion is based on the age of the different clades that belong to each of these three categories according to the dated phylogeny of Breitkopf et al. (2015). Ophrys speculum, which is exemplary of category one,

\footnotetext{
${ }^{4}$ An invalid synonym of Ophrys speculum is $O$. ciliata
} 
is among the most ancient clade according to this phylogeny. The clades of Ophrys fusca and $O$. sphegodes, to which belong the species of the third category, are the youngest clades in which speciation is recent and rapid. According to our suggestion, the visual signals proposed in this latter category are still perfectible by directional selection.

Moreover, all molecular phylogenies produce so far concur to show that the floral morphology leading to abdominal copulation attempt is a synapomorphy shared by all the species belonging to the former, paraphyletic Pseudophrys subgenus (e.g. Breitkopf et al. 2015, Bateman et al. 2018a). To explain how this synapomorphy arose, we assume the apparition of a deviant morphology in flowers of species belonging to one of the four basic clades of the phylogeny (Figure 1). This new morphological type provoked either by mutations of genes controlling flower morphogenesis and flower hairiness, or by hybridization between taxa from distant clades with contrasted morphologies, or by a combination of the two would have targeted more efficiently the abdomen of the pollinators towards the retinaculum and the stigmatic cavity compared to flowers on which males moved randomly. Next, the higher reproductive success of those flowers that possess these more efficient pollinator guides could have led to the spread of this key innovation, and favor the rapid radiation in this group demonstrated by phylogenomic analyses (Breitkopf et al. 2015, Joffard et al. in review) by de novo speciation and/or hybridization.

\subsubsection{Directional selection on flowers of recently separated species}

The assumption of directional selection on flowers after speciation can be tested on couples of species that are recently separated. The clade of $O$. insectifera that presents a basal divergence within Ophrys according to the dated molecular phylogeny of Breitkopf et al. (2015) seems an excellent candidate to test this assumption. In this clade, the widely distributed $O$. insectifera parent species is associated to two endemic seemingly recently separated vicariants (Devey et al. 2008, Breitkopf et al. 2015). The basic, less parsimonious hypothesis is thus that the most widespread species is the parent of its endemic vicariants, which by the way forms sympatric populations with populations of the two vicariants. These vicariants are $O$. subinsectifera that is restricted to the southern foothill of the central and eastern Pyrenees and $O$. aymoninii that is endemic to the calcareous part of Massif Central in southern France. These three species have their own pollinators that are phylogenetically distinct Hymenopterans (i.e. wasps, solitary bees and sawflies) of contrasted size (Table 3, Figure 5). These pollinators are belonging to three insect families, which is a unique case within the Ophrys genus. Molecular insights from cpDNA sequencing and amplified fragment length polymorphisms genotyping indicate a recent diversification in the clade of $O$. insectifera, which may have been further obscured by active migration and admixture across the European continent (Triponez et al., 2013). Genetic results still indicate weak but noticeable phylogeographic clustering that correlates only partially with species limits. Moreover, several isolated haplotypes and genetic clusters were reported for $O$. insectifera in central and southeastern Europe, which might favor the speciation of other endemics in this clade (Triponez et al., 2013). The flowers of the three species show remarkable similarities in shape and coloration, notably an elongated labellum with a blue-grey horizontal bar in its middle. Their main originalities relative to other Ophrys flowers are the form of the labellum showing three well-delineated lobes, two laterally and one basally, the latter being subdivided in two parts, the elongation and the shrinkage of the petals, and the location of the pseudo-eyes on the labellum and not on the stigmatic cavity (Devillers \& Devillers-Terschuren 1994). 
Table 3. Pollinator identity and body size (body length in $\mathrm{mm}$ ) of the species of the $O$. insectifera clade. Data from various sources compiled in Triponez et al. (2013).

\begin{tabular}{|c|c|c|}
\hline Fly orchid species & Main pollinator species (order and family) & Pollinator size \\
\hline Ophrys insectifera & Argogorytes mystaceus (and A. fargei) (Hymenoptera ; Crabronidae) & $9.5-12$ \\
\hline Ophrys subinsectifera & Sterictophora gastrica (Hymenoptera ; Argidae) & $6.8-7.2$ \\
\hline Ophrys aymoninii & Andrena combinata (Hymenoptera ; Andrenidae) & $8.5-9.5$ \\
\hline
\end{tabular}

Altogether, these flowers show a remarkable convergence towards an insect body with the petals mimicking the antennae, the stigmatic cavity mimicking the head, the anterior lobes mimicking the wings, and the rest of the labellum mimicking the thorax and the abdomen (Figure 2).

Nonetheless, careful examination and measurements of the flowers revealed differences between these three taxa. The labellum of $O$. insectifera is very usually devoid from yellow border, whereas flowers of $O$. subinsectifera have a yellow outer border ranging from 1 to $1.5 \mathrm{~mm}$, and those of $O$. aymoninii from 1 to $2.5 \mathrm{~mm}$ (Triponez et al. 2013). The length of the petals of $O$. subinsectifera is shorter $(2-4 \mathrm{~mm})$ than those of the two other species $(4-7 \mathrm{~mm})$. More importantly, the length of the labellum of the three species is significantly different (ca. $12 \mathrm{~mm}$ for 0 . insectifera, $9.5 \mathrm{~mm}$ for $O$. aymoninii and $6.5 \mathrm{~mm}$ for $O$. subinsectifera, Triponez et al. 2013). This difference in labellum length matches the non-overlapping body lengths of the three pollinators (Table 3 ).

Following our speciation scenario mentioned before, and under the assumption of the anteriority of the widespread species, new pollinators would have been attracted by locally divergent individuals of $O$. insectifera that randomly crossed a peak of their olfactory landscape to escape intraspecific competition for limited, mnemonic pollinators. Accordingly, the comparison of labellum extracts from $O$. insectifera and $O$. aymoninii showed that on the five GC-EAD organic compounds that were found to be physiologically active for males of Andrena combinata (the pollinator of $O$. aymoninii), four were in significantly higher relative amount in O. aymoninii (Gervasi et al. 2017). The application of these four compounds on flowers of $O$. insectifera triggered approach and landing of males of $A$. combinata (Gervasi et al. 2017). Moreover, no pollinia transfer between these two taxa was recorded in a field experiment using artificially stained pollinia, whereas pre- and post-zygotic barriers are absent as shown by the success of interspecific artificial pollinia transfer leading to fruit set and seedling development (Gervasi et al. 2017).

The couple $O$. insectifer $-O$. aymoninii seems thus well isolated by those differences in floral scents. Next, following our speciation scenario, directional selection on flower traits would have taken place. The differences in flower shape and colors, together with the match in size observed between the pollinators and their respective flower labellum meets this prediction. The difference in labellum length would be a decisive character to maintain the isolation between the two taxa by limiting or preventing cross fecundation. The couple $O$. insectifer $-O$. subinsectifera seems less advanced on the route of speciation. The flowers of $O$. subinsectifera emit three aldehydes that are absent from the bouquets of the two other species (Joffard et al. 2016). Unfortunately, we are lacking data on their physiological activity. Also, we are lacking data on the existence of other preand post-zygotic isolation mechanisms other than pollinator attraction. However, careful observations and pictures by Paulus (2017) document that the males of Sterictophora gastrica (the 
pollinator of $O$. subinsectifera) are attracted by sympatric individuals of $O$. insectifera. This suggests an incomplete olfactory isolation, with the existence of divergent $O$. insectifera individuals that are still able to attract Sterictophora gastrica. When $O$. insectifera is in sympatry with the two other Ophrys species, some very rare hybrids are observed in both cases (B. Schatz unpublished data).

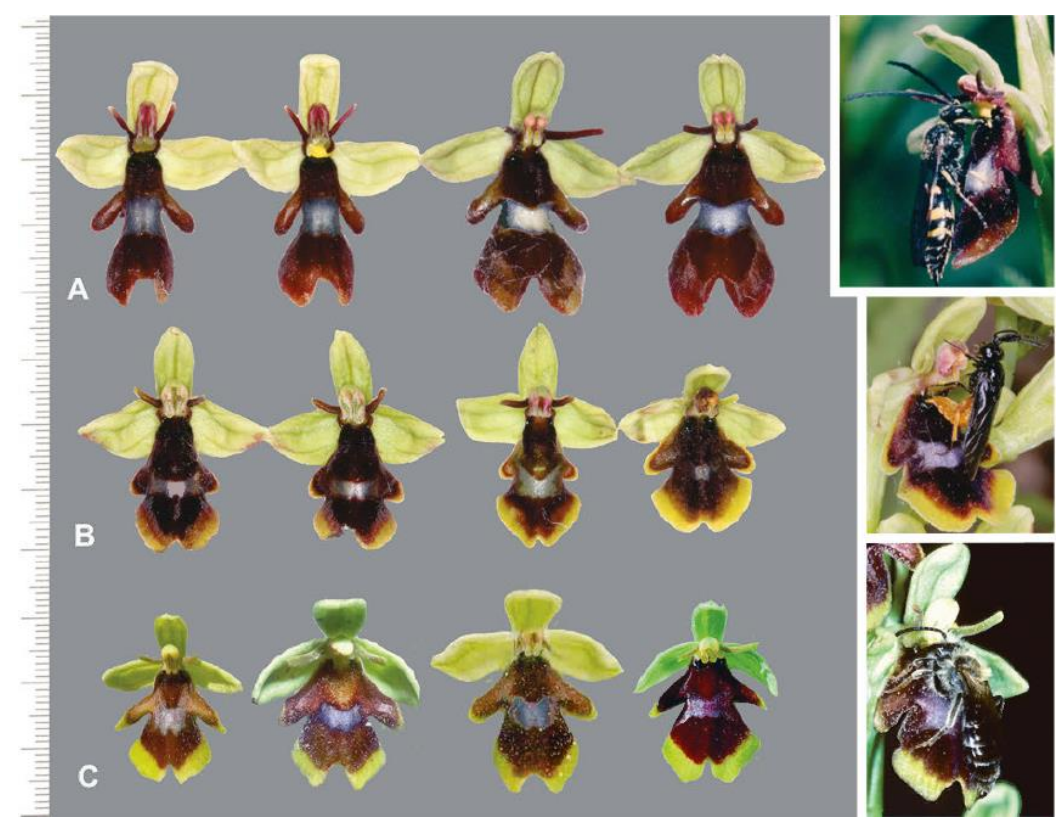

Figure 5. The flowers of the three species of the Ophrys insectifera clade and their pollinators. A. Ophrys insectifera and Argogorytes mystaceus. B. Ophrys subinsectfera and Sterictophora gastrica. C. Ophrys aymoninii and Andrena combinata. Picture reprinted from Paulus 2017, with permission.

Nonetheless, a directional selection leading to differences in flower traits should already have taken place, leading to the differentiation of $O$. subinsectifera, as revealed by differences in flower size, shape and colors. The reverse situation (i.e. the attraction of males of Argogorytes sp. by $O$.

aymoninii) has not been reported so far; copulation attempts between these partners would be very difficult due to the difference in size between the large pollinator and the small labellum of the flower. As previously mentioned, the pollinator of $O$. subinsectifera is a sawfly, which is an exception in Ophrys. Another exception is the posture used by this pollinator during pollinia transfer. Rather than trying to perform a copulation attempt with the flower either in cephalic or abdominal position, sexually excited males of Sterictophora gastrica move frenetically on the flower and pick up pollinia on their thorax, their legs or the side of their abdomen (Souche 2007, Geniez et al. 2016, Paulus 2017). This frenetic behavior corresponds to the prediction of Ågren et al. (1980), who wrote that "a chemical stimulus without the proper tactile stimulation only results in undirected crawling and flattering of the wings of the males". This particularity is another argument in favor of a very recent differentiation of $O$. subinsectifera, and we suggest that a progressive evolution of flower morphology, and particularly hairiness would increase the efficiency of pollination of $O$. subinsectifera flowers by Sterictophora gastrica. Besides, the behavior of males of Sterictophora gastrica provides insights on the evolution of the particular morphology of flowers pollinated by males attempting to copulate using the abdominal position. 


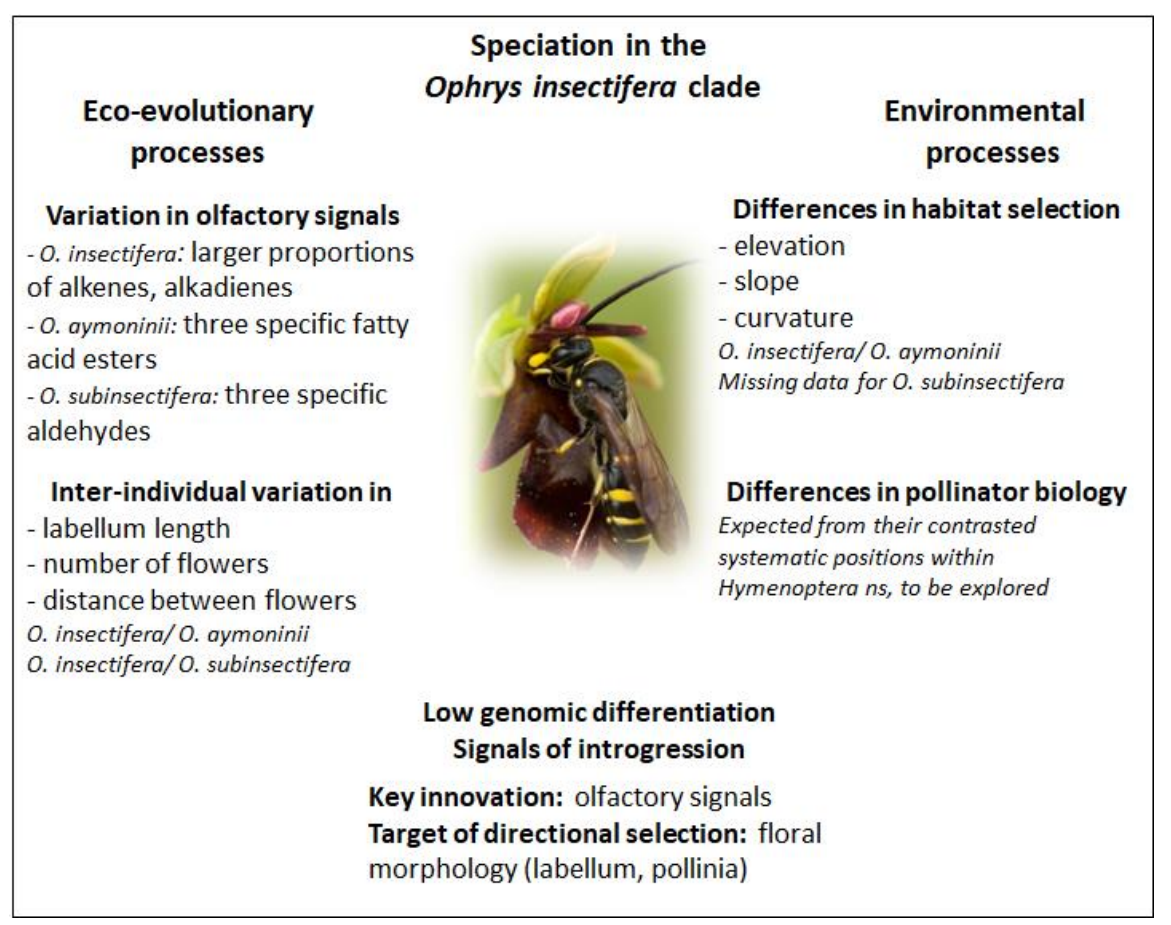

Figure 6. Current knowledge about the potential speciation drivers in the Ophrys insectifera clade (photo @ S. Witzthum). Based on Triponez et al. 2013, Joffard et al. 2016.

Altogether, the investigation of differences in flower shape, size and colors in the $O$. insectifera clade provides us with a glimpse on the evolutionary processes that are at work on flower morphology after a pollinator shift (Figure 6). As anticipated by Devillers \& Devillers-Terschuren (1994), this clade is an excellent candidate to get insights on speciation in Ophrys. Obviously, more experimental works are needed to deepen these insights along 4 research axes:

(1) to test for differences in flower odor and morphology among $O$. insectifera individuals that are either in sympatry or in allopatry with both vicariants, to phenotype hybrids, and to investigate whether flower shape, and especially the labellum length, will favor or impede pollinia removal in con- and inter-specific cross fecundation experiments;

(2) to investigate carefully the habitat selection of the three taxa in relation with the habitat requirements of their pollinators;

(3) to test whether the different genetic clusters evidenced in Ophrys insectifera could correspond to ongoing speciation towards new endemics using genotypic and phenotypic data together with data on habitat requirements and pollinator identity;

(4) to investigate if the identity of the two main pollinators of $O$. insectifera (roughly one in the southern part and the other in the northern part of its range) induces a pollinatormediated selection on the olfactory signals emitted by the flowers.

\subsubsection{Directional selection on flowers of the same species using two different pollinators}

The recent review of the orchid-pollinator network in the Euro-Mediterranean region offered several potential cases in which one Ophrys species is pollinated by two or several pollinators (Joffard et al. 2019). However, investigations considering main and secondary pollinators are still rare, which often precludes a reliable separation of these two categories in the actual state of knowledge of Ophrys pollination biology. We can tentatively generalize that the use of two different pollinator species is observed in Ophrys species either with a wide, continuous distribution range like the above 
mentioned Ophrys insectifera, or with a discontinuous range like in taxa potentially isolated on islands. Even if understudied, such insular situations provide a unique opportunity to investigate how allopatric speciation interacts with pollinator shifts. In a nutshell, different metapopulation lineages of the same parent taxon attracting two or more pollinator species should engage on the road of speciation through directional selection on species-specific pollinator signals. Such speciation events will be facilitated by the disruption of gene flows between incipient species that occurs in allopatry. Accordingly, we expect to find situations of spatially disjoint metapopulation lineages at various stages of the speciation process, which begins by the attraction of different pollinators by morphologically similar individuals. These situations of ongoing speciation show the interest of the unified species definition, according to which species diversification is a continuous process. Breitkopf et al. (2013) documented that in the Italian peninsula, an Adriatic population of $O$. sphegodes exclusively attracted $A$. nigroaenea, which is considered as the legitimate pollinator of the species, whereas a Tyrrhenian $O$. sphegodes population preferentially attracted the pollinator bee Andrena bimaculata. Both populations are separated by $>200 \mathrm{~km}$ and by the Apennine Mountaines chain. This pollinator shift was associated with significant differences in scent component proportions. However, using neutral markers (AFLP), the authors did not detect significant intraspecific genetic structuring between these two populations. These results suggest that these two populations have reached an early stage of divergence and are adapting to different pollinator species.

Allopatric speciation is evident on the five main islands of the Mediterranean Sea (Sicily, Sardinia, Corsica, Cyprus, Crete), which all have several endemic and sometimes closely related species (e.g. Delforge 2016). The presence of endemic is also recorded on certain other islands like Malta or Hvar, but speciation on island occurred mainly in the Aegean Sea (Karpathos, Andros, Astypalea or Rhodes (Delforge 2016, Schatz 2017). The presence of Ophrys species is strongly limited in very small islands where a sustainable, long-term presence of their pollinators is uncertain (Schatz 2017). However there are numerous situations in which closely related Ophrys species are present on two, three or a few more islands: they could be considered as an ideal experimental design to test the our scenario of speciation by pollinator shift by the progressive evolution of pollinator attraction signals. Such situations are currently understudied, mainly for logistical reasons.

Besides pollinator displacements in allopatry, some studies report the use of several related pollinator species in the same locality. Paulus (2018) mentions that two species closely related to the main pollinator of Ophrys sphegodes (Andrena nigroaenea) participate to its pollination (A. thoracica in France and A. limata in SW Germany). He suggests that such sharing of the pollination function occurs when there is no competition among Ophrys species for a given pollinator species, which requires a kind of control of the efficiency of its pollinator(s) by the plant. To support this interesting hypothesis, Paulus (2018) reports a case study on Ophrys fuciflora in which flowers are visited almost at the same rate by the main pollinator Eucera longicornis and by the closely related E. nigrescens. The latter species revisit Ophrys flowers much more rarely, probably due to an imperfect correspondence between the bouquet of organic compounds emitted by the flowers and the sexual pheromone of $E$. nigrescens virgin females. Paulus (2018) suggests that mnemonic males of $E$. nigrescens associates this imperfect odor bouquet with a vain copulation attempt, and avoid further contacts with the flowers of $O$. sphegodes after their first trial. We can also interpret this avoidance by suggesting that the variations in the odor bouquet produced by flowers of $O$. sphegodes are not sufficiently different for $E$. nigrescens males, so that they consider all other flowers as already visited 
after a first copulation attempts. Whatever their reason E. nigrescens males thus "steal" pollinia instead of performing pollination. Paulus (2018) points out that the flowering period of $O$. fuciflora coincides with the emergence of fresh males of E. longicornis at a time when E. nigrescens males that emerged earlier become rare and older, which might be an adaptation to cope with this waste of pollinia by the less effective pollinator. We can thus expect over time an always closer match between a plant and the best pollinator species, either by directional selection on the flowering period, or by directional selection on the organic compounds emitted by the flower and the pheromones of its best pollinator female. Such always closer matches, sometimes associated to postzygotic barriers could explain why there are not even more bee-orchid species produced by hybridization. Yet, we have to keep in mind that this match can be relaxed according to the local and spatio-temporal availability of the main pollinator.

\subsubsection{Directional selection on species sharing the same pollinator}

When two species of Ophrys species share the same pollinators, we anticipate directional selection leading to convergence in some floral traits to secure pollinator attraction, but divergent selection on some other floral traits to limit or avoid hybridization. A nice example of these antagonistic processes has been reported in the couple Ophrys normanii - $O$. chestermanii that was extensively studied by Gögler and collaborators (Gögler et al. 2009, 2011, 2015). Both species are endemic from Sardinia where sympatric and allopatric populations coexist. Both species share the same pollinator, the male of the parasitic bumblebee Bombus vestalis, which removes the pollinia on his head (copulation attempts in cephalic position). These two species are paraphyletic according to genetic analyses using both amplified fragment length polymorphism and plastid markers (Gögler et al. 2009). This is concordant with the dated molecular phylogeny of Breitkopf et al. (2015). These species are indeed considered to belong to distant lineages, $O$. normanii being a member of the rather primitive clade formed around $O$. tenthredinifera, whereas $O$. chestermanii belongs to the more recent clade represented by Ophrys umbilicata in the phylogeny of Breitkopf et al. (2015). However, the flowers of both species show a remarkable convergence in shape, color and hairiness. In particular, the flowers of both species have black stigmatic cavities and black basic fields, whereas no other species in their respective clade has these peculiarities.

Odorless dummy bees impregnated with labellum extracts from both species attract males of Bombus vestalis that perform copulation attempts with the dummies in flight cage experiments (Gögler et al. 2009). Using GC-MS and GC-EAD, Gögler et al. (2011) found that the bouquet of organic compounds produced by the flowers of both species were remarkably similar to the pheromones of virgin females of Bombus vestalis. Given this similarity of the floral odor bouquet, the overlap of their flowering periods and the co-occurrence of both species in sympatric populations, hybridization between these taxa is expected. Genetic assignment of 99 individuals of $O$. chestermanii and of 65 individuals of $O$. normanii failed to detect gene flow between the two species (Gögler et al. 2009). Moreover, interspecific cross-pollination experiments showed that 11 out of 15 crosses with $O$. chestermanii as pollen donor resulted in fruits, and 13 of 15 crosses with 0 . normanii as pollen donor resulted in fruits. In all but one case, seeds of these cross-pollination combinations germinated, and produced 100-150 plantlets from each combination after 18 months (Gögler et al. 2009).

Given the absence of gene flow and of post-zygotic isolation despite a remarkable similarity in the composition of the odor bouquet emitted by the flowers, we expect here the existence of an efficient pre-zygotic isolation mechanism. Careful measurements of flower morphology were 
performed on individuals from both species sampled in sympatric and allopatric populations (20 flowers/population, 8 to $20 \mathrm{~km}$ between sympatric and allopatric populations). These measurements concerned three floral traits that are involved in the pollination process: the width and the height of the stigmatic cavity and the length of the pollinaria, and a fourth trait, the length of the central outer tepal, which is not involved in the pollination syndrome, was added to serve as control. Results indicate unambiguously that the values of the three florals traits associated with pollination were significantly less similar between the two species in the population in sympatry compared to their values in allopatric populations, whereas no difference was detected on the control trait. The stigmatic cavity of $O$. chestermannii individuals was smaller in the population where both species were in sympatry. The pollinaria of $O$. normannii were significantly longer than those of $O$. chestermannii. This situation led Gögler et al. (2015) to postulate that the long pollinaria of $O$. normannii fixed on the head of males of $B$. vestalis would not fit into the smaller stigmatic cavity of $O$. chestermannii; this was confirmed by video observations of copulation attempts. The differences in those floral traits that are associated with pollination in sympatric population relative to allopatric populations suggest a displacement of characters leading to pre-zygotic isolation between these two phylogenetically distinct taxa, which nicely fits the hypothesis of a divergent selection on floral traits to avoid hybridization.

This example shows that directional selection leads not only to convergent evolution towards flowers with similar morphology, but also to refined mechanisms that allow pre-zygotic isolation by mechanically preventing cross-pollination between flowers sharing similar visual signals of attraction for the pollinator's viewpoint. The presumption of character displacement in sympatric populations suggests that the design of such pre-zygotic mechanisms is under strong selective pressure. Given the experimental demonstration of the absence of post-zygotic mechanisms leading to the production of hybrid seedlings combined with the lack of observation of flowering hybrids in the field such a selection for the evolution of pre-zygotic mechanisms suggests hybrid mortality at later stage.

\subsection{Exploitation of protandry and the resulting directional selection on Ophrys flowering period}

Consistent with protandry, most Ophrys pollinators (adult males of solitary bees) usually emerge before their females (Alcock et al. 1978, Hutchings et al. 2018). The theory of protandry suggests that in insects in which females mate only once and males are capable of multiple mating, male development time is under strong intra-sexual selection, as those males that are active and sexually mature at the time of virgin female emergence will maximize their mating success (Wiklund \& Fagerström 1977, Courtney \& Parker 1982, Zonneveld \& Metz 1991). Accordingly, it would be interesting for plants using pollination by sexual swindle to bloom when male competition is at a peak, i.e. when there is a relative deficit of virgin females (Nilsson 1992). This appealing hypothesis contrasts with the longevity of flowers in Ophrys, even we acknowledge the paucity of published data on this critical biological parameter. Francisco \& Ascensão (2013) mention periods of anthesis of 6-8 days and 9-12 days for Ophrys bombyliflora and 0 . tenthdedinifera, respectively, whereas Neiland \& Wilcox (1995) observed that unpollinated flowers of $O$. tenthredenifera and $O$. arachnitiformis remained intact in the field for three weeks or more. As each Ophrys inflorescence carries several flowers that open successively, albeit with a slight overlap between subsequent flowers, a given Ophrys individual with a mean of four flowers should present at least one attractive flower during $c a$. 4-6 weeks. We can conclude from the long duration of the offer of attractive flowers that the plants 
are not able to predict correctly the time window of the emergence of their pollinators. This can be due to the unpredictability in space and time of the emergence of pollinators. Even if it is possible to compute in the lab the required degree-day accumulation for solitary bee emergence (e.g. White et al. 2009), there is a strong micro- and meso-climatic heterogeneity leading to a quite large time window during which their emergences do occur (Thomson \& White 2011). Moreover, holometabolous insects have the possibility to delay their adult emergence according to the ambient, prevalent weather conditions for days or even weeks (e.g. Hermann et al. 2016). This delay is another hinder for the use of precise degree-day accumulation as predictor of pollinator apparition. Finally, historic data of both the flowering dates of Ophrys sphegodes and the flight periods of its pollinator (Andrena nigroaenea) over more than 350 years, provided a very interesting and rare evidence that Ophrys flowering periods become more and more imperfectly adjusted to the development time of their pollinator under the effect of global warming (Hutchings et al. 2018). As the rate of development of bees increases faster with increasing temperature than that of Ophrys (Robbit et al. 2014), the interval between the male flight period and the flowering time of Ophrys increased. As the interval between the male and the female flight period of the bee decreased with warming, the proportion of females increased in the population before Ophrys flowering, which is likely to lower the attractive power of flowers to naïve males (Robbit et al. 2014). Consequently, current climate warming induced a progressive time shift between pollinator emergence and Ophrys flowering, which decreases Ophrys pollination probability because bee females are on their wing at the same time, or even before anthesis.

Whatever its reason, the exploitation of protandry and hence the long period during which a given Ophrys individual has flowers at anthesis is another factor that might facilitate the random crossing of olfactory peaks leading to the attraction of a new species of pollinator. Adults of a given species of solitary bee are usually on the wings during 8-12 weeks a year, but these weeks cover a period ranging from February to September if we consider for instance all the species belonging to the Andrena genus in the UK (Williams \& Edwards 2012, Else \& Edwards 2018). Accordingly, once some flowers within the same metapopulations attract a new pollinator species and interbreed, we can expect a directional selection for a more precise match between the flowering period of this newly formed Ophrys taxon and the flight period of its pollinator. The end result of this directional selection would be the staggered flowering periods of closely related Ophrys species attracting different species of closely related pollinator species.

A good example is provided by the West-Mediterranean Ophrys fusca clade pollinated by males of solitary bees belonging to the Andrena genus (Paulus 2006). It is worth noticing here that in this group, which is considered as of recent origin by molecular phylogenies (Breitkopf et al. 2015, Joffard et al. in review), the morphological differentiation of Ophrys species seem rather weak, whereas the temporal segregation of the flowering period of syntopic species is well established. The Table 4 shows the flowering periods of a suite of very similar species of the Ophrys fusca clade belonging to the Ophrys attaviria group (sensu Delforge 2016) that are all found in Crete. All these species are pollinated by different Andrena species and their flowering period is remarkably staggered from the beginning of January to the end of May. Only one of these species (Ophrys cinereophila) is widely distributed in Crete and in the Eastern part of the Mediterranean basin, whereas the others are very rare and restricted to the different calcareous mountain massifs in Crete. This situation fits well an incipient speciation scenario in which species differentiation based on a new pollinator attraction within metapopulations is followed by a directional selection on flowering 
period leading to a progressive divergence from the parent taxon, whereas morphological changes did not yet occur.

Table 4. Flowering periods (months of the year) of species of the Ophrys attaviria group from Crete and their Andrena pollinators. Here, the three first species are isolated from the three last because of the absence of overlap period in their respective flowering period. Data from various sources including Delforge (2016), Paulus (2018).

\begin{tabular}{|c|c|c|c|c|c|c|}
\hline & & \multicolumn{5}{|c|}{ Flowering period } \\
\hline Ophrys & Andrena & I & II & III & IV & v \\
\hline O. siatica & A. nigroaenea & & & & & \\
\hline O. creticola & A. bimaculata & & & & & \\
\hline O. thriptiensis & A. bicolor & & & & & \\
\hline O. cinereophila & A. cinereophila & & & & & \\
\hline O. cressa & A. merula & & & & & \\
\hline O. creberrima & A. creberrima & & & & & \\
\hline O. pallidula & A. combinata & & & & & \\
\hline O. kedra & A. variabilis & & & & & \\
\hline
\end{tabular}

Indeed, we anticipate that the interbreeding between individuals attracting by chance a new pollinator will occur locally, in such a way that newly differentiated taxa had smaller distribution ranges. Such narrow distribution ranges might thus be used to identify recent speciation events.

\subsection{Pollinator repulsion after fecundation}

Fecundation in Ophrys, i.e. the deposit of pollinia on the stigmatic cavity of a flower, is often associated to the removal of a pollinia from this newly pollinated flower by the pollinator (e.g. Schiestl \& Ayasse 2001). Accordingly, the prolonged maintenance of the attractiveness of a pollinated flower will have a low additional reproductive reward for the plant. We thus expect the selection of mechanisms limiting this attractiveness, especially when the individual has other, unpollinated flowers at anthesis that will benefit from the visit of a pollinator.

\subsubsection{Emission of anti-aphrodisiac compounds}

Recently pollinated Ophrys flowers emit anti-aphrodisiac compounds that are reluctant for pollinator males, similar to those pheromones that are produced by mated Hymenopteran females to avoid further courtships. Schiestl et al. (1997) found an alteration in the olfactory signals produced by the flowers, both by the decrease of the total amount of produced organic compounds, and by a change in their composition, which favor some repellent compounds that reach high proportions in the whole bouquet. The key role of an organic compound (the fatty ester all-trans-farnesyl hexanoate) was later on demonstrated in the repulsion of pollinators after flower pollination by Schiestl \& Ayasse (2001). These authors compared the organic organic compounds present in the head spaces and in labellum extracts of unpollinated flowers and of flowers three ( 2 to 4 ) days after pollination. They found a decrease, albeit non-significant, of elecrophysiologically active compounds in labellum extracts of pollinated flowers, but, more importantly, a significant increase in both the absolute and in the relative amount of all-trans-farnesyl hexanoate in the headspace of pollinated flowers. This fatty ester produced by pollinated flowers is also emitted by females of the pollinator of Ophrys sphegodes (the solitary bee Andrena nigroaenea) after mating (Schiestl \& Ayasse 2000); females use this ester to line their brood cells. In bioassays, bee dummies impregnated by attractive scents of unmated females combined with farnesyl hexanoate elicit significantly less approach and copulation attempts by males than dummies impregnated by attractive scents only (Schiestl \& Ayasse 2000). Similarly, the presentation of flowers of Ophrys shegodes artificially scented with an amount of 
farnesyl hexanoate equal to the increased amount after pollination lowers the number of pollination attempts by male bees and were thus significantly less attractive than flowers treated with solvent only (Schiestl \& Ayasse 2001). This finding led these authors to suggest that the emission of this ester by pollinated flowers is a signal that will facilitate visits by the pollinator to other flowers of the same inflorescence. However, such successive visits could expose the plant to geitonogamy, i.e. the pollination of a flower by another flower of the same inflorescence, which has similar consequences than autogamy in terms of decrease in seed production and viability. To counter these deleterious outcomes of geitonogamy, careful observations by Ayasse et al. (2000) revealed a subtle mechanism. In this species, pollination can be achieved only if the pollinia carried by the bee on his head from one flower have bend forward to contact the stigmatic surface of the next flower visited. The time of complete bending of pollinia (161.9 $\pm 10.1 \mathrm{~s}, \mathrm{n}=10$, Claessens \& Kleynen 2011) is longer than the total time spent by males to visit the first, second, and third flower of an inflorescence, and to move between these flowers (Ayasse et al. 2000). Altogether, this exemplary study constitutes so far the only documented example in Ophrys of the modification of communication signals between the flower and its pollinator induced by pollination. Even though this single case study deserves generalization, it provides many insights on the eco-evolutionary dynamics between Ophrys and their pollinators.

\subsubsection{Repulsion by learning visual signals}

We have mentioned before that pollinator attraction involves the combination of olfactory and visual signals. Pollinator repulsion by Ophrys flowers seems also use these two channels. Schiestl et al. (1997) reported that the colors of pollinated flowers of Ophrys sphegodes faded away three days after pollination. Gaskett (2011) suggested that varying floral shape could impair pollinator learning avoidance in the same way that scent variation hinders this process. Using an elegant experimental approach, Stejskal et al. (2015) went a step further in deciphering the function of the complex drawings on the labellum (i.e. the macula) of the flowers of Ophrys heldreichii. These drawings form complex and variable whitish patterns on a dark brown background. Field observations reveal that after a copulation attempt, males of its pollinators (the bee Eucera berlandi) fly at close distance in front of the flower during ca. 1 minute. The authors interpret this behavior as a scan by the bee of the drawing of the macula, which should impair further copulation attempts on the same flower, and even on flowers of the same inflorescence. Indeed, comparisons of labellum patterns revealed that patterns within inflorescences are more similar than those of other conspecific plants. The authors hypothesize that the ultimate function of the drawings of the macula for the plant would thus be the avoidance of geitonogamy. According to this hypothesis, this mechanism based on individual drawing learning should be less efficient than the emission of anti-aphrodisiac compounds as in Ophrys sphegodes, because here after a pollination attempt pollinators are deterred from visiting other flowers of the same inflorescence. To test whether the pollinators could have the capacities to learn individual drawings, Stejskal et al. (2015) trained honeybees (Apis mellifera) and showed that trained individuals were able to discriminate among labellum drawings coming from different plants, but not among labellum drawings coming from the same inflorescence. These results support the hypothesis that the variable labellum drawings of $O$. heldreichii are involved in flower-pollinator communication, which would likely help these plants to avoid geitonogamy. 


\section{The romance from a pollinator viewpoint: repulsion, deception or benefit?}

As previously mentioned, almost all Ophrys species use pollination by sexual swindle, i.e. flowers mimic signals of virgin females of their pollinators to elicit copulation attempts by males that ensure pollinia transfer. Observations of this behavior led to the introduction in the literature of the concept of "pollination by sexual deceit, according to which the plant is considered as acting in a devious way by parasitizing male sexual behavior (e.g. Vereecken 2009). If we apply a cost-benefit analysis of the consequences of the Ophrys pollination syndrome from the pollinator viewpoint, costs are indeed evident. Duped males attracted to Ophrys by the manipulation of combined olfactory, visual and tactile signals will lose time and energy in copulation attempts with flowers. Some data exist on the duration of the copulation attempt by male pollinators. In the couple Ophrys leocchroma -Eucera kullenbergi, Rakosy et al. (2017) investigated the relation between the duration of the copulation attempts and its efficiency as estimated by the number of massulae that were deposited in the stigmatic cavity. The relation was significantly positive, with copulation attempt durations ranging from $5 \mathrm{sec}$ to $60 \mathrm{sec}$. In the couple Ophrys galilea - Lasioglossum marginatum, Machaka-Houri et al. (2018) recorded a mean duration of $14 \mathrm{sec}$. In the couple Ophrys heldreichii - Eucera berlandi, Stejskal et al. (2015) reported a median duration of $5.7 \mathrm{sec}$, ranging from $0.7 \mathrm{sec}$ to $39.9 \mathrm{sec}$. This duration is congruent with the amount of time required for the successful transfer of sperm in honey bees (a matter of seconds, Koeniger \& Koeniger 1991, Winston 1991) or in stingless bees (less than a minute, Engels \& Engels 1988). Indeed, copulation entails costs (Brown \& Baer 2005), i.e. an enhanced exposure to predation because copulating pairs have a greatly reduced mobility (Brown \& Baer 2005), and hence are more conspicuous. In two Australian Cryptostylis orchids that use a pollination syndrome by sexual swindle similar Ophrys, males of their shared pollinator (the wasp Lissopimpla excelsa, Ichneumonidae) do not only attempt to copulate with flowers mimicking their female lures, but they also ejaculate on the flowers, as demonstrated by the presence of sperm in their stigmatic cavity (Gaskett et al. 2008). To our best knowledge, the presence of insect sperm on the labellum of Ophrys flowers was never reported so far. We ignore if this question has ever been investigated, and it certainly deserves attention.

Even if there is no formal proof right now that pollinators of Ophrys complete to its very end the full sequence of their mating behavior with Ophrys flowers, we have elements to challenge to some extent the concept of "pollination by sexual deceit". We agree with Vereecken (2009) that Ophrys flowers parasitize the mating behavior of their pollinators by mimicking more or less faithfully the signals emitted by Hymenopterans virgin females. However, we advocate that the semantic choice of the words "deceit" or "deception" to qualify this pollination syndrome reflects an anthropogenic representation of the mental state of the pollinator rather than the biological reality. Indeed, the usual justification of the "deception" of the male is his effort to memorize the identity of the flower with which he attempted to mate, to avoid losing time and energy in another dupery by the same flower. We hope to have demonstrated above that this memorization is a normal event in the sequence of the male mating behavior, which was selected to avoid a loss of time and energy spent in courting a previously mated female. In a nutshell, the pollination syndrome of Ophrys is based on sexual swindle of males by female lures, but there is no evidence that pollinators are deceived. Instead, antenna grooming or visual scanning of the drawings of the macula for olfactory or visual signals respectively (Paulus 2006) that occur after a copulation attempt with an Ophrys 
flower might correspond to the normal end of the mating behavior for satisfied Hymenopteran males that record the identity of their partner.

We hypothesize that pollinator insects could even benefit from Ophrys flowers for both habitat matching and dispersal. Ophrys flowers emit female-like species-specific sexual pheromones that fix pollinator males within suitable habitats where potential mates might be available for reproduction. Female Hymenopterans produce pheromones that travel over large distances but the speed and the directionality of such olfactory cues are poor. Males in search of females are thus confronted to a highly discontinuous signal of rapidly fluctuating concentrations of olfactory signals (Svensson et al. 2014). By increasing locally the concentration of pheromone-like molecules, Ophrys flowers might contribute to the location by their pollinators of suitable habitats within complex landscapes, which provides insects with an increase of their male and female reproductive success. Besides, by repulsing pollinators after copulation attempts, Ophrys flowers might favor male dispersal, which limits the previously mentioned inbreeding risks to which populations of haplodipoïd Hymenopterans are particularly exposed. We are not aware of data supporting this hypothesis in Ophrys. In the Australian orchid Drakaea glyptodon that uses a similar pollination by sexual swindle, observations and mark and recapture experiments suggest that pollinators (males of the Tiphiidae wasp Zaspilothynnus trilobatus) immediately leave the area after visiting a flower and do not visit nearby flowers within a refractory period (Peakall 1990).

\section{Molecular bases of adaptive radiation in Ophrys}

We gathered so far many evidences supporting the scenario of co-evolutionary dynamics between Ophrys and their pollinators, and hence advocating that the adaptive radiation in Ophrys is promoted by intraspecific competition for mnemonic pollinators. This radiation is characterized by a large intraspecific variability in flower olfactory signals mimicking insect sexual pheromones. This variability is driven by the need to avoid pollinator habituation, itself generated by the ability of pollinators to recognize and memorize the identity of their mates on basis of the composition of their bouquet of sexual female-like pheromones. Accordingly, the key innovation that favors the adaptive radiation in Ophrys is the variability in female-like sexual pheromones produced by the flowers that can induce pollinator shift. Although we continuously accumulate knowledge on the ecoevolutionary drivers of this impressive adaptive radiation, the molecular bases of the striking phenotypic variation displayed by Ophrys spp. remain poorly understood. For example, the functional role of MADS-box genes that are key players of flower development in plants has never been really investigated in Ophrys. The difficulty to elucidate the causes of phenotypic variation in Ophrys has been delayed by their relatively high-sized genome ( 10 billions of base pairs: Leitch et al., 2009, Abreu et al., 2017), which has hindered the feasibility of genomic investigations in this genus. Like in other biological systems, this limitation is being overcome by the increasing development of high throughput sequencing technologies, especially considering that the Ophrys genome size may actually rather be of 5-7 Gbps in most diploid species (Bou Dagher-Kharrat et al., 2013, J. Bertrand, unpublished data, P.M. Schlüter, personal communication). The amount of genomic resources available for Ophrys is currently still limited to the three published plastid genomes of $O$. iricolor, O. sphegodes and O. aveyronensis (Roma et al. 2018; Bertrand et al. 2019). Hereafter, we will speculate on the mechanisms that would allow phenotypic changes, emphasizing on changes in the organic organic compounds composing the floral olfactory phenotypes 
We mentioned above that candidate gene approaches have revealed the involvement of particular homologs of the stearoyl-ACP (acyl carrier protein) desaturases ('SADs') such as SAD2 and $S A D 5$ in flower variations of organic organic compounds that could explain the attraction of different pollinator and reproductive isolation between closely related Ophrys species (see Schlüter et al. 2011, Xu et al. 2012, Sedeek et al. 2016). In the example of $O$. sphegodes and $O$. archipelagi detailed above, two mutations in the sequence coding for SAD5 increased the attractive power of the olfactory signals produced by the former species (Sedeek et al. 2016). Such genetic changes in a smattering of key genes reinforce the idea that the fast speciation in radiating Ophrys is genic rather than genomic (Wu 2001, Wu \& Ting 2004) and depends on a few genes coding for those traits that are responsible for pollinator attraction. This view is corroborated by the pioneering study of Sedeek et al. (2014) that was the first, and so far, the only one to have used a genomic approach (Genotyping-BySequencing, GBS) to compare patterns of differentiation of the genomes of four closely related, sympatric and co-flowering Ophrys species (O. archipelagi, O. incubacea, O. garganica and $\mathrm{O}$. shegodes). Although this method sampled a relatively small fraction of the high-sized Ophrys genome, the authors found that only a very small proportion $(<0.05 \%)$ was repeatedly identified as more strongly differentiated between pairs of species than expected. This finding is in good agreement with a speciation process beginning with changes (mutations and/or gene expression modifications) occurring in a few key genes which would precede the genome-wide differentiation stages and confirms the interest of such biological systems to identify those loci that are prominently involved at early stages of the evolutionary divergence. Such 'blind' approach highlighted new candidate genes and advantageously complement 'classical' candidate gene approaches. Among the 'few' outlier markers of species differentiation identified by Sedeek et al. (2014) using GBS, three loci are located in annotated genes: Vacuolar Protein Sorting 45 (VPS45; AT1G77140.1 and ECERIFERUM 1 (CER1; $1 T 1 G 02205.3)$ known to be or putatively involved in flower odor production in plants. We detailed above the case of the two desaturase genes SAD2 and SAD5 that modulate the production of different alkenes responsible for the attraction or repulsion of two different pollinators, which led to the sympatric speciation of Ophrys sphegodes and $O$. archipelagi. Although the former example relies on a 'classical' mutation scheme provoking amino acid change, the molecular mechanisms involved in such changes may rely not only on physical alteration of DNA coding sequences. Changes in the relative concentration of the organic organic compounds in the olfactory signals would be easily achieved by up- or down-expressing the genes coding for enzymes catalyzing reactions leading to the assemblage of these molecules.

In spite of these convincing examples explaining differences in Ophrys flower odor production, we are far to understand mechanisms prone to explain the magnitude and rapidity of whole phenotypic variation at the scale of the genus Ophrys. An increasing number of studies report that the reprogramming of gene expression by transposable elements insertions plays a key role in the adaptive evolution of plants (Lisch 2013, Seidl \& Thomma 2016). Relevant to Ophrys adaptative radiation is the finding that transposable elements movements may cause very rapid programmatic changes and dramatically modify the phenotype. For example, the embedded machinery that ensure the 'copy-and-paste' mechanism of replication of long terminal repeat (LTR) retrotransposons is responsible for the expression of previously inactive coloration genes, that change the colour of the fruit in some grapevine, orange or apple varieties (see Lisch 2013; Zhang et al., 2019). Thus, transposable elements can produce variation at a rate that can vary by orders of magnitude in a few generations, and this rate can be responsive to exactly the changes in environmental conditions that 
can impose strong selection pressures (Lisch 2013). Therefore, transposable elements provide a rich source of genetic and regulatory diversity between individuals, thereby contributing to the adaptive evolution of their plant hosts to novel environments (Seidl \& Thomma 2016). Moreover, it has been shown that plants display structured genomes with transposable-element-rich regions that mediate accelerated evolution (Seidl \& Thomma 2016).

We hypothesize that genes coding for enzymes catalyzing reactions leading to the organic organic compounds found in the odor bouquet of Ophrys flowers might reside in such regions where gene expression is highly variable. This location could explain the huge variability observed in both biologically active and inactive organic organic compounds that is recorded in the flower odor bouquets. Moreover, the clustering of the genes that are responsible for variation in odor emission in such regions means that reproductive isolation, and hence some speciation events, could at least initially be driven by pure epigenetics mechanisms. This original scenario of sympatric speciation by epigenetics mechanism is in good agreement with the current impossibility of detecting inter-specific genetic variation between many Ophrys species with methods based on DNA sequencing of short fragments of a fraction of the whole genome to detect mutations, like RADSeq (Bateman 2018, Bateman et al. 2018a). Also, the location within regions of accelerated evolution of the genes responsible for individual variation in odor bouquet means that speciation in Ophrys depends more on changes in a few key genes rather than on genomic-wide processes (Sedeek et al. 2014). Moreover, such a scenario of epigenetics control of speciation could explain why the speciation rate is so fast in this radiating genus. In this vein, the evidence that populations of cells located in the labellum of Ophrys flowers are polyploïd (Bateman et al. 2018b) opens a new research front in the quest for the mechanisms involved in Ophrys speciation. In five species of Ophrys belonging to five different clades in the phylogeny of Breitkopf et al. (2015) and including the basal and the more derived clades, Bateman et al. (2018b) showed that the peripheral area of the labellum margin, which is rich in unicellular elongated trichomes, presented a wide spectrum of polyploidy cells. Some of these trichomes were octoploid, meaning that their nuclei had undergone two cycles of endoreplication. No or only a weak evidence of polyploidy was found in other parts of the labellum (the speculum and the appendix, respectively). Bateman et al. (2018b) suggest that there could be an epigenetic control of the overexpression of genes via the local induction of nucleus endoreplication in particular tissues. They mention that the overexpression of genes induced by such a highly localized endoreplication could induce change in the odor bouquet emitted by Ophrys flowers, and thus play a role in pollinator attraction and pollinator shift.

Aside, reassembly of ancient genetic variation into new combinations has been proposed as another explanation of the paradox of rapid speciation rates in radiating lineages despite slow mutation rates (Marques et al. 2019). We mentioned before examples supporting that hybridization is for sure a cause of speciation in Ophrys. Within the high number of described hybrids, it is possible to find crossings even among species belonging to the different clades identified in the phylogeny of Breitkopf et al. (2015). This means that genome admixtures occur at each Ophrys generation, which has the potential to create hybridogenetic species. There are only but a few studies that used molecular markers to investigate the level of introgression or hybridization between Ophrys species. Aiming at assessing the real effect of hybridization in diversification, Cotrim et al. (2016) investigated the genetic structure of Ophrys fusca s.I. and $O$. lutea using nuclear and plastid microsatellite markers. They found that up to $12 \%$ of the genotyped plants were misclassified according to their floral phenotypes. However, these individuals did not cluster into a new group, which could indicate 
introgression and gene flow between these "parent" species. Cotrim et al. (2016) mention that "distinguishing hybrid introgression from common ancestry and incomplete lineage sorting remains a critical task in evolutionary studies". Admittedly, Ophrys fusca s.I. and O. lutea belongs to the same clade according to a time-calibrated phylogeny of 19 species based on three nuclear genes (Joffard et al. in press), two of which coding for traits involved in pollinator attraction (pheromone-like molecule metabolism and flower development). This evidence of a co-ancestry origin of the two study species is contrary to the premises of Cotrim et al. (2016), and questions the putative hybridogenetic origin of these misclassified individuals.

A more robust genetic evidence of the existence of hybridogenetic species is provided by the existence of polyploïd species. Polyploïdy can occur through hybridization between existing species, and induce the immediate apparition of reproductive isolation, a prerequisite for adaptive evolutionary divergence. Amich et al. (2007) reported the presence of 6 polyploïd species in the Iberian Peninsula, all belonging to the section Pseudophrys. Interestingly, polyploidy within Pseudophrys appears to be restricted to the Western Mediterranean Basin, and may have contributed to the speciation of this section in the Iberian Peninsula, with five endemic or geographically restricted species. Polyploidy is associated with phenomena of hybrid sterility and changes in gene expression that are observed between polyploid species from hybrid origin and their diploid parents. These changes occur through both gene silencing and the activation of new genes (e.g. Soltis et al. 2004, Schoenfelder \& Fox 2015), which might facilitate the acquisition of reproductive isolation by modifying the expression of genes coding for traits associated with the attraction of a new pollinator.

\section{New research avenues}

Our discovery of the fascinating functioning of Ophrys was accompanied by the emergence of multiple questions. Here, rather than a fastidious chronological enumeration of those research topics that appeared successively during our review, we propose a transversal approach to some new avenues that might provide decisive breakthrough in the study of adaptive radiation. However, we summarize all research questions in Figure 7. A first transversal question overarching all our review is the relative role of epigenetics and genetics mechanisms in Ophrys speciation. We suggest addressing this issue by investigating the consistency of floral phenotypes over time. To what extent floral phenotypes produced by a given individual will remain stable both within year and from one year to the next? From a mechanical viewpoint, mounting evidences show that transposable elements can induce gene silencing or gene overexpressing in plants facing biotic stresses (Seidl \& Thomma 2017). It would be interesting to investigate if and how the experience of an individual might influence its phenotype over its lifetime in long-lived plants such Ophrys. This question includes first the consistency of the bouquets of their pollinator female-like pheromone over time. If we admit that changes in gene expression is key in the intra-specific variation of the floral odor bouquet, we can speculate that a given individual could alter its female-like pheromone profile both within year and between years according to its current (within-year) or former (between year) reproductive success. 


\section{Knowledge to acquire}

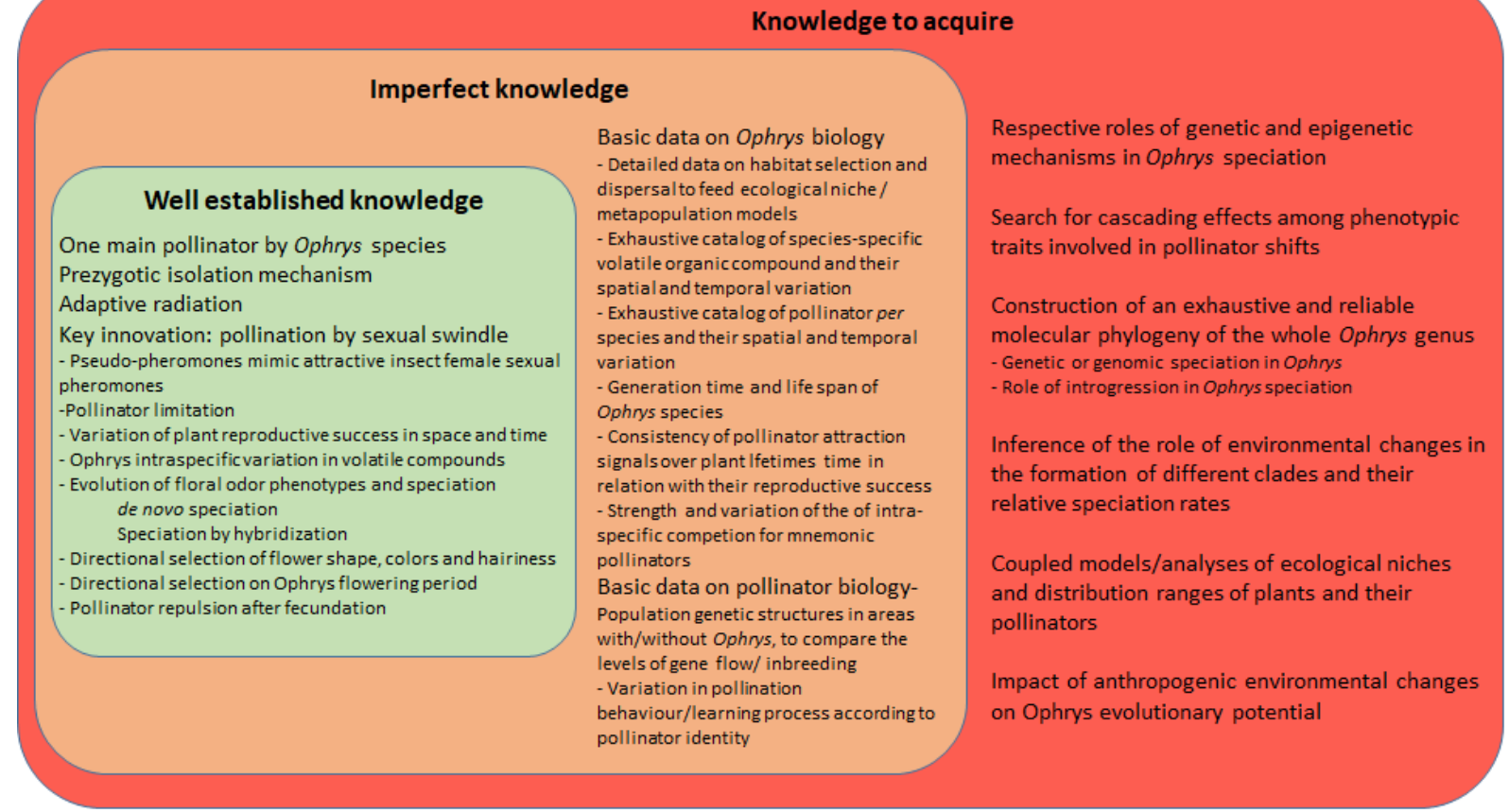

Figure 7. General overview of the states of knowledge about Ophrys speciation, with emerging research questions.

We can speculate further that in the absence of fecundation, the odor bouquet produced by the flowers will progressively shift from altering the concentration of substances attracting the legitimate pollinator (the biologically active EAD organic compounds mentioned above) to recruiting new molecules, and hence resulting in the formation to a brand new odor cocktail susceptible to attract a new pollinator. The same temporal consistency holds true for the other components of the phenotypes but here rather between years. Also, it should be exciting to look for cascading effects among phenotypic traits, i.e. to what extent changes in olfactory signals are associated to variation in other components of the phenotypes. Even if our enthusiast hypothesis about changes in floral signals over time according to the previous experience of the plant individual would be falsified, this latter point is worth investigating and will provide insights on the possible existence of a pollination syndrome potentially driven by epigenetics mechanisms. To tackle this issue, we suggest the adoption of an integrate framework linking molecular and cellular investigations, by considering nonlinear and interactive relationships (including feed-backs) among all 'omic' compartments (Baguette et al. 2015) and by comparing the expression of genes coding for those traits that responsible for pollination attraction over time in cells from vegetal (leaves) and reproductive (flowers) tissues.

A second issue is the construction of an exhaustive and reliable molecular phylogeny of the whole Ophrys genus. Exhaustive because the most used and useful one (the work of Breitkopf et al. 2015 ) is based on less than $10 \%$ of the species currently known. Reliable because given the low differentiation of the Ophrys genome, it should be based on the use of an important number of loci, each probably containing a limited amount of information. Those loci should harbour flanking regions that would be conserved enough among Ophrys species to be amplified, but should be also as variable as possible to be differentiated among clades and species (see Loiseau et al., 2019, for a recent example of the use of baits protocol). Besides providing a definitive response to the endless and sterile controversy about species definition in Ophrys, this phylogeny would provide insights on the genetic material that is available for potential speciation events based on epigenetics 
mechanisms, if any. Accordingly, this phylogeny based on DNA sequence could be compared to phenotypic data to disentangle the relative roles of phylogenetic constraints and of floral evolution in the evolution of floral phenotypes (Joffard et al. in press for a seminal analysis of this topic). The availability of an exhaustive and reliable molecular phylogeny based on DNA sequences would also provide insights on the history of the radiation, which is of particular interest to detect rapidly evolving clades. Using detailed genomic data on the Orchidaceae family that are now available to calibrate the radiation in Ophrys, it would be possible to infer more precisely the role of environmental changes in the formation of different clades and their relative speciation rate.

Besides, this molecular phylogeny based on DNA sequences would allow the comparison of the distribution range and the metapopulation dynamics of parent, ancient and derived, recent taxa. The idea here is to combine data collected across different scales of biological organization, from individual features to species distribution range, through population and metapopulation dynamics. Accordingly, the reproductive success of individuals belonging to parent and derived species will be monitored over time and related to individual genotype at traits involved in pollinator attraction and phenotypic variability. Besides, the reproductive success will be related to population features, like distance to the closest neighbor, pollinator abundance, conspecific density, the presence and density of congeneric species, or habitat shape and area. Such data should be collected in several metapopulations over time, to generate an integrate data set linking individual fitness to population and metapopulation dynamics in spatially-, phenotypically- and genetically- explicit contexts, and compared to predictions of ecological niche models and to the real distribution of the respective species. This procedure should provide first-hand data on the speed of new species expansion and insights on the advantages of the colonization of competitor-free space. Finally, combined with similar data on the pollinators, such comparisons will provide detailed insights on the best way to conserve the evolutionary potential of the genus in the current context of anthropogenic global change. We particularly encourage to a collaborative work using similar approaches and protocols among the different scientific teams working in the Mediterranean region.

A third issue concerns the coevolution between plants and their pollinators. We mention that pollinators could benefit from the presence of Ophrys for both habitat matching and dispersal. To our best knowledge, there are no conclusive data to support this hypothesis. This issue might also be addressed experimentally, by recording in closed arenas the behavior of pollinators confronted to their legitimate females or to those Ophrys flowers that they use to visit. Clever observations of the males could inform on the respective effects of females and flowers on the propensity of the male to stay in the arena or to leave, which corresponds to habitat matching and dispersal, respectively. Besides, these experiments allow the test of the repulsion or deception hypothesis mentioned above, by comparing the consistency of pollinator behavior during copulation with their legitimate females and during copulation attempts with flowers. Those Hymenopteran males that have a similar behavior in both treatments should be considered as being not duped; conversely, if the post-mating behavior of males differs between the treatments, we should expect that he associated the copulation with a flower to some costs. It would be interesting to perform such experiments with both parent and derivate plants as inferred from a dated molecular phylogeny, to investigate to what extent the plant-pollinator relationship could reinforce over time since the pollinator shift responsible for speciation. 


\section{Conclusions}

1. Bee-orchids (Ophrys sp.) use pollination by sexual swindle, i.e. their flowers mimic signals emitted by receptive females of insects (mainly solitary bees). This highly specialized pollination strategy led to the evolution of striking morphological similarities between flowers of bee orchids and the females of their species-specific pollinators.

2. The understanding of the fundamental role of the olfactory signals in the functioning of pollination by sexual swindle triggered a profusion of studies disentangling the relationships between Ophrys flowers and their pollinators. Combined field and lab works showed similarities of olfactory signals in derived Ophrys species sharing the same pollinator species, or differences between closely related Ophrys species with different pollinator species. Resolving the phylogeny of Ophrys should have been a priority to be able to clearly identify (groups) of related species and to consider their evolutionary relationships with their pollinators. However, a consensual Ophrys phylogeny is still lacking because the very definition of what is a species of bee-orchid is vigorously debated. To summarize, two main species concepts are in conflict: a definition of species based on DNA sequence homologies, and a definition of species based on prezygotic isolation by attraction of species-specific pollinators. According to the first definition, there should be currently around ten species of bee orchids, whereas the adoption of the prezygotic isolation criterion would lead to the recognition of several hundred of species.

3. The unifying species definition validates the prezygotic isolation criterion that considers the existence of several hundreds of Ophrys species. Given the recent origin of this genus $\left(<5.10^{6} \mathrm{yr}\right)$, this explosion of species on such a sort time lapse corresponds to a radiation. We tried to highlight the processes by which this radiation occurred, and to what extent it was adaptive. We found that the current hypothesis that explains speciation in Ophrys, i.e. negative frequency-dependent selection was not corresponding to the high intra-specific variability existing in the olfactory signals used by Ophrys flowers to attract species-specific pollinators. Instead, we found a battery of independent evidences showing that the high speciation rate in Ophrys occurred through intraspecific competition between plants competing for pollinators that have high cognition and memorization abilities. Indeed, Hymenopterans pollinators developed individual mate recognitions both to avoid mating with kin and to loose time and energy to court previously mated females. Flowers of Ophrys plants must thus differ from each other to be attractive to their mnemonic pollinators. Accordingly, intraspecific competition would generate a large number of distinct olfactory phenotypes, which is in agreement with field and lab observations, but which disagrees with the prediction of the negative frequency-dependent selection hypothesis that predicts the existence of only but a few phenotypes.

4. This variation of olfactory phenotypes could by chance drive random crossings of peaks in the olfactory landscape of the pollinator guild that is syntopic to each particular Ophrys population and thus attract a new pollinator species. This event could lead to the prezygotic isolation of a new taxon if it occurred simultaneously in several plants within the same population, and is usually followed by a directional selection on flower morphology that will reinforce the attraction of the new pollinator.

5. The pollination strategy by sexual swindle of bee-orchids may be considered as a key innovation triggering an adaptive radiation by intraspecific competition for mnemonic pollinators. This radiation is adaptive because the newly isolated Ophrys taxa benefit from a competitor-free space. This finding 
reshaped and unified the voluminous scientific literature dealing with Ophrys into a common and integrated eco-evolutionary framework, which generated several exciting research avenues about (1) the molecular mechanisms involved in adaptive radiations, (2) the relative roles of sympatric and allopatric speciation in in adaptive radiations and (3) the asymmetric plant-pollinator coevolution that could be at work in this particular adaptive radiation.

\section{Acknowledgments}

We thank for financial supports the OSU-Oreme that funded long-term observations and the GDR Pollineco $N^{\circ} 2058$ (CNRS-MTES). Michel Baguette, Joris Bertrand and Virginie M. Stevens are members of the Excellence Lab TULIP (ANR-10-LABX-41). Michel Baguette acknowledges the CHANGE team of the Theoretical and Experimental Ecology Station of the National Center for Scientific Research (CNRS) for insightful comments. J. Claessens and H. F. Paulus and S. Witzthum kindly provided the rights to use pictures 2,5 and 6 , respectively. 


\section{References}

Ågren L., Kullenberg B., Sensenbaugh T. 1984. Congruences in pilosity between three species of Ophrys (Orchidaceae) and their hymenopteran pollinators. Nova Acta Regiae Societatis Scientiarum Upsaliensis Serie V 3, 5-25

Alcock J., Barrows E. M., Gordh G., Hubbard L. J., Kirkendall L., Pyle D.W., Ponder T.L., Zalom F. G. 1978. The ecology and evolution of male reproductive behavior in the bees and wasps. Zoological Journal of the Linnean Society 64, 293-326

Althoff D.M., Segraves K., Johnson M.T.J. 2014. Testing for coevolutionary diversification: linking pattern with process. Trend in Ecology and Evolution 29, 82-89

Amich F., Garcia-Barriuso M., Bernardos S. 2007. Polyploidy and speciation in the orchid flora of the Iberian Peninsula. Botanica Helvetica 117, $143-157$

Arditti J., Ghani A.K.A. 2000. Numerical and physical properties of orchid seeds and their biological implications. New Phytologist 145, 367-421

Armbruster W.S. 2017. The specialization continuum in pollination systems: diversity of concepts and implications for ecology, evolution and conservation. Functional Ecology 31, 88-100

Ayasse M. 2006. Floral scent and pollinator attraction in sexually deceptive orchids. In N. Dudareva, E. Pichersky (Eds.), Biology of floral scent. CRC, Boca Raton, 219 - 241

Ayasse M., Schiestl F., Paulus H.F., Lofstedt C., Hansson B., Ibarra F., Francke W. 2000. Evolution of reproductive strategies in the sexually deceptive orchid Ophrys sphegodes: how does flower-specific variation of odor signals influence reproductive success? Evolution 54, 1995-2006

Ayasse M., Paxton R.J., Tengö J. 2001. Mating behavior and chemical communication in the order Hymenoptera. Annual Review of Entomology 46, 31-78

Ayasse M., Schiestl F.P., Paulus H.F., Ibarra F., Francke W. 2003. Pollinator attraction in a sexually deceptive orchid by means of unconventional chemicals. Proceedings of the Royal Society Series B $270,517-522$

Ayasse M., Gögler J., Stökl J. 2010. Pollinator-driven speciation in sexually deceptive orchids of the genus Ophrys. In: M. Glaubrecht (Ed.), Evolution in Action, 101-116. Springer, Berlin

Baguette M., Legrand D., Stevens V.M. 2015. An individual-centered framework for disentangling genotype-phenotype interactions. Trends in Ecology and Evolution 30, 709-711

Baguette M., Michniewicz, R.J. Stevens V.M. 2017. From genes to metapopulations. Nature Ecology and Evolution 1, 0130

Barrows E.M., Bell W.J., Michener C.D. 1975. Individual odor differences and their social functions in insects. Proceedings of the National Academy of Sciences 72, 2824-2828

Bateman R.M., Bradshaw E., Devey D.S., Glover B.J., Malmgren S., Sramkó G., Thomas M.M., Rudall P.J. 2011. Species arguments: clarifying competing concepts of species delimitation in the pseudocopulatory orchid genus Ophrys. Botanical Journal of the Linnean Society 7, 336-347

Bateman R.M. 2018. Two bees or not two bees? An overview of Ophrys systematics. Berichte aus den Arbeitskreisen Heimische Orchideen 35, 5-46

Bateman R.M., Sramkó G., Paun O. 2018a. Integrating restriction site-associated DNA sequencing (RAD-seq) with morphological cladistic analysis clarifies evolutionary relationships among major species groups of bee orchids. Annals of Botany 121, 85-105

Bateman R.M., Guy J.J., Rudall P.J., Leitch I.J., Pellicer J., Leitch A.R. 2018b. Evolutionary and functional potential of ploidy increase within individual plants: somatic ploidy mapping of the complex labellum of sexually deceptive bee orchids. Annals of Botany 122, 133-150 
Beaumont H.J.E., Gallie J., Kost C., Ferguson G.C., Rainey P.B. 2009. Experimental evolution of bet hedging. Nature 462, 90-93

Bertrand J.A.M, Gibert A., LLauro C., Panaud O. 2019. Characterization of the complete pastime of Ophrys aveyronensis, a Euro-Mediterranean orchid with an intriguing disjunct geographic distribution. Mitochondrial DNA Part B, in press.

Blondel J., Aronson J. 1999. The Mediterranean region: biological diversity in space and time. Oxford University Press, Oxford

Borg-Karlson A.K. 1990. Chemical and ethological studies in the genus Ophrys (Orchidaceae). Phytochemestry 29, 1359-1387

Borràs J., Cursach J. 2018. Analysis of the parameters that affect the reproductive success of Ophrys balearica P. Delforge. In: Pons G.X., del Valle, L., Vicens D., Pinya, S., McMinn M., F. Pomar (Eds.), Llibre de ponències i resums de les VII Jornades de Medi Ambient de les Illes Balears, 41-44. Societat d'Història Natural de les Balears (SHNB) - Universitat de les Illes Balears (UIB).

Bou Dagher-Kharrat M., Abdel-Samad N., Douaihy B., Bourge M., Fridlender A., Siljak-Yakovlev S., Brwon S.C. 2013 Nuclear DNA C-values for biodiversity screening: case of the Lebanese flora. Plant Biosystems 147, 1228-1237

Breitkopf H., Schlüter P.M., Xu S., Schiestl, F.P., Cozzolino S., Scopece G. 2013. Pollinator shifts between Ophrys sphegodes populations: might adaptation to different pollinators drive population divergence? Journal of Evolutionary Biology 26, 2197-2208

Breitkopf H., Onstein R.E., Cafasso D., Schlüter P.M., Cozzolino S. 2015. Multiple shifts to different pollinators fuelled rapid diversification in sexually deceptive Ophrys orchids. New Phytologist 207, 377-389

Brisson D. 2018. Negative frequency-dependent selection is frequently confounding. Frontiers in Ecology and Evolution 6, 10

Brown M.J.F., Baer, B. 2005. The evolutionary significance of long copulation duration in bumble bees. Apidologie 36, 157-167

Byers K.J.R.P., Xu S., Schlüter P.M. 2017. Molecular mechanisms of adaptation and speciation: why do we need an integrative approach? Molecular Ecology 26, 277-290

Charlesworth C., Charlesworth D. 1987. Inbreeding depression and its evolutionary consequences. Annual Review of Ecology and Systematics 18, 237-267

Chen, C., Song, Q., Proffit, M., Bessière, J. M., Li, Z. \& Hossaert-McKey, M. 2009 Private channel: a single unusual compound assures specific pollinator attraction in Ficus semicordata. Functional Ecology 23, 941-950

Claessens J., Kleynen J. 2011. The flower of European orchid: form and function. Schrijen-Lippertz, Voerendaal

Claessens J., Kleynen J. 2016. Orchidées d’Europe. Fleurs et pollinisation. Biotope, Mèze

Condamine F.L., Clapham M.E., Kergoat G.J. 2016. Global patterns of insect diversification: towards a reconciliation of fossil and molecular evidence? Scientific Reports 6, 19208

Correvon H., Pouyanne M.A. 1916. Un curieux cas de mimétisme chez les Ophrydées. Journal de la Société Naturelle Horticole de France 4, 29-47

Correvon H., Pouyanne M.A. 1923. Nouvelles observations sur le mimétisme et la fécondation chez les Ophrys speculum et lutea. Journal de la Société Naturelle Horticole de France 4, 372 - 377 
Cortis P., Vereecken N. J., Schiestl F. P., Barone Lumaga, M. R. Scrugli, A., Cozzolino S. 2009. Pollinator convergence and the nature of species' boundaries in sympatric Sardinian Ophrys (Orchidaceae). Annals of Botany 104, 497-506

Cotrim H., Monteiro F., Sousa E., Pinto M.J., Fay M.F. 2016. Marked hybridization and introgression in Ophrys sect. Pseudophrys in the western Iberian Peninsula. American Journal of Botany 103, 677-691

Cuervo M., Rakosy D., Martel C., Schulz S., Ayasse M. 2017. Sexual deception in the Eucera-pollinated Ophrys leochroma: a chemical intermediate between wasp- and Andrena-pollinated species. Journal of Chemical Ecology 43, 469-479

Danforth B.N., Cardinal S., Praz C., Almeida E.A.B., Michez D., 2013. The impact of molecular data on our understanding of bee phylogeny and evolution. Annual Review of Entomology 58, 57-78

Darwin C. 1859. On the origin of species by means of natural selection, or the preservation of favoured races in the struggle for life. John Murray, London

de Queiroz K. 2005. Different species problem and their resolution. Bioessays 27, 1263-1269

de Queiroz K. 2007. Species concepts and species delimitation. Systematic Biology 56, 879-886

Delforge P. 2005. Un pollinisateur pour Ophrys bombyliflora. Les Naturalistes Belges 18, 91-94

Delforge P. 2016. Orchidées d'Europe, d'Afrique du Nord et du Proche Orient. 4th edition. Delachaux et Niestlé, Lausanne

Delle-Vedove R., Schatz B., Dufa》M. 2017. Understanding intraspecific variation of floral scents in the light of evolutionary ecology. Annals of Botany 120, 1-20

Devey D. S., Bateman R. M., Fay M.F., Hawkins J. A. 2008. Friends or relatives? Phylogenetics and species delimitation in the controversial European orchid genus Ophrys. Annals of Botany 101, 385402

Devillers P., Devillers-Terschuren J. 1994. Essai d'analyse systématique du genre Ophrys. Les Naturalistes Belges 75, 273-400

Devillers P., Devillers-Terschuren J. 2013. Orchidées et concepts modernes de l'espèce. Les Naturalistes Belges 94, 61-74

Dormont L., Joffard N., Schatz B. 2019. Intraspecific variation in floral color and odor in orchids. International Journal of Plant Sciences (in press)

dos Santos C.F., Imperatriz-Fonseca V.L., Arias M.C. 2016. Relatedness and dispersal distance of eusocial bee males on mating swarms. Entomological Science 19, 245-254

Ehrlich, P.R. and Raven P.H. 1964. Butterflies and plants: a study in coevolution. American Naturalist, $18,586-608$

Eickwort G.C., Ginsberg H.S. 1980. Foraging and mating behavior in Apoidea. Annual Review of Entomology 25, 421-446

Else G.R., Edwards M. 2018. Handbook of the bees of the British Isles. The Ray Society, London

Engels E., Engels W. 1988. Age dependent queen attractiveness for drones and mating in the stingless bee Scaptotrigona postica. Journal of Apicultural Research 27, 3-8

Fabre J.H. 1852. De la germination des Ophrydées et de la nature de leurs tubercules. Annales des Sciences Natutelles Botanique Série IV 5, 163-186

Fabre J.H. 1924. Souvenirs entomologiques. Delagrave, Paris

Ferdy J.B., Gouyon P.H., Moret J., Godelle B. 1998. Pollinator behavior and deceptive pollination: learning process and floral evolution. American Naturalist, 152, 696-705 
Ferdy J.B., Austerlitz F., Moret J., Gouyon P.H., Godelle B. 1999. Pollinator-induced density dependence in deceptive species. Oikos 87, 549-560

Ferton Ch. 1923 La vie des abeilles et des guêpes. Chiron, Paris

Forrest J.R.K., Thomson J.D. 2011. An examination of synchrony between insect emergence and flowering in Rocky Mountain meadows. Ecological Monographs 81, 469-491

Fortel L., Henr, M., Guirao A.L., Kuhlmann M., Mouret H., Rollin O., Vaissière B.E. 2014. Decreasing abundance, increasing diversity and changing structure of the wild bee community (Hymenoptera: Anthophila) along an urbanization gradient. PLoS One 9, e104679

Francisco A., Ascensão L. 2013. Structure of the osmophore and labellum micromorphology in the sexually deceptive orchids Ophrys bombyliflora and Ophrys tenthredinifera (Orchidaceae). International Journal of Plant Sciences 174, 619-636

Galán Cela P., Seligrat I., Ortúñez E., Gamarra R., Vivar A., Scrugli A. 2014. Un estudio de la micromorfología de las semillas en el género Ophrys (Orchidaceae). Anales del Jardino Botanica de Madrid 71, e008

Gandon S. 1999. Kin competition, the cost of inbreeding and the evolution of dispersal. Journal of theoretical Biology 200, 345-364

Gaskett A. 2011. Orchid pollination by sexual deception: pollinator perspectives. Biological Reviews 86, 33-75

Gaskett A.C., Winnick C.G., Herberstein M.E. 2008. Orchid sexual deceit provokes ejaculation. American Naturalist 171, E206 - E212

Gavrilets S., Losos J.B. 2009. Adaptive radiation: contrasting theory with data. Science 323, 732-737

Geniez P., Schatz B., Escudié P., 2016. Confirmation of the presence of Ophrys subinsectifera Hermosilla \& Sabando in France. L'Orchidophile 210, 213-217

Gervasi D.L., Schiestl F.P. 2017. Real-time divergent evolution in plants driven by pollinators. Nature Communications 8, 14691

Gervasi D.L., Selosse M.A., Sauve M., Francke W., Vereecken N.J., Cozzolino S., Schiestl F.P. 2017. Floral scent and species divergence in a pair of sexually deceptive orchids. Ecology and Evolution 7, 6023-6034

Gigord, L. D., MacNair, M. R., Smithson, A. 2001. Negative frequency dependent selection maintains a dramatic flower color polymorphism in the rewardless orchid Dactylorhiza sambucina (L.). Soo. Proceedings of the National Academy of Sciences 98, 6253-6255

Gögler J., Stökl J., Sramkova A., Twele R., Francke W., Cozzolino S., Ayasse M. 2009. Ménage à trois two endemic species of deceptive orchids and one pollinator species. Evolution 63, 2222-2234

Gögler J., Twele R., Francke W., Ayasse M. 2011. Two phylogenetically distinct species of sexually deceptive orchids mimic the sex pheromone of their single common pollinator, the cuckoo bumblebee Bombus vestalis. Chemoecology 21, 243-252

Gögler J., Stökl J., Cortis P., Beyrle H., Barone Lumaga M.R., Cozzolino S., Ayasse M. 2015. Increased divergence in floral morphology strongly reduces gene flow in sympatric sexually deceptive orchids with the same pollinator. Evolutionary Ecology 29, 703-717

Gomulkiewicz R., Drown D.M., Dybdahl M.F., Godsoe W., Nuismer S.L., Pepin, K.M., Ridenhour, B.J., Smith C.I., Yoder J.B. 2007. Dos and don'ts of testing the geographic mosaic theory of coevolution. Heredity, 98, 249-258.

Grant P.R., Grant B.R. 2006. Evolution of character displacement in Darwin's finches. Science 313, 224-226 
Hairston N.G., Ellner S.P., Geber M.A., Yoshida T., Fox J.A. 2005. Rapid evolution and the convergence of ecological and evolutionary time. Ecology Letters 8, 1114-1127

Hanksi I. 1999. Metapopulation Ecology. Oxford University Press, Oxford

Heimpel G.E., De Boer J.G. 2008. Sex determination in the Hymenoptera. Annual Review of Entomology, 53, 209-230.

Hembry D.H., Yoder J.B., Goodman, K.R. 2014. Coevolution and the diversification of life. American Naturalist, 184, 425-438

Hermann S.L., Xue S., Rowe L., Davidson-Lowe E., Myers A., Eshchanov B., Bahlai C.A. 2016. Thermally moderated firefly activity is delayed by precipitation extremes. Royal Society Open Science 3, 160712.

Hutchings M.J. 2010. The population biology of the early spider orchid Ophrys sphegodes Mill. III. Demography over three decades. Journal of Ecology 98, 867-878

Hutchings M.J., Robbirt K.M., Roberts D.L., Davy A.J. 2018. Vulnerability of a specialized pollination mechanism to climate change revealed by a 356-year analysis. Botanical Journal of the Linnan Society 186, 498-509

Hutchinson G.E. 1959. Homage to Santa Rosalia or why are there so many kinds of animals. American Naturalist 93, 145-159

Jacquemyn H., Brys R., Waud M., Busschaert P., Lievens B. 2015. Mycorrhizal networks and coexistence in species-rich orchid communities. New Phytologist 206, 1127-1134

Jaquemyn H., Hutchings M.J. 2015. Biological Flora of the British Isles: Ophrys sphegodes. Journal of Ecology 2015, 1680-1696

Joffard N., Buatois B., Schatz B. 2016. Integrative taxonomy of the fly orchid group: insights from chemical ecology. The Science of Nature, 103, 77

Joffard N., Massol F., Grenié F., Montgelard C., Schatz B. 2019. Effect of pollination strategy, phylogeny and distribution on pollination niches of Euro-Mediterranean orchids. Journal of Ecology $107,478-490$

Joffard N., Arnal V., Buatois B., Schatz B., Montgelard C. in press. Floral scent evolution in the section Pseudophrys: phylogenetic constraints or pollinator-mediated selection?

Johnson S.D., Nilsson L.A. 1999. Pollen carryover, geitonogamy, and the evolution of deceptive pollination systems in orchids. Ecology 80, 2607-2619

Koeniger N., Koeniger G. 1991. An evolutionary approach to mating behaviour and drone copulatory organs in Apis, Apidologie 22, 581-590

Kullenberg B. 1961. Studies in Ophrys pollination. Zoologiska bidrag från Uppsala. 34, 1-340.

Kullenberg B., Bergström G. 1976. Hymenoptera Aculeata males as pollinators of Ophrys orchids. Zoologica Scripta 5, 13-23

Lamichhaney S., Berglund J., Almén M.S., Maqbool K., Grabherr M., Martinez-Barrio A., Promerova M., Rubin C.J., Wang C., Zamani N., Grant B.R., Grant P.R., Webster M.T., Andersson L. 2015.

Evolution of Darwin's finches and their beaks revealed by genome sequencing. Nature 518, 371-375

Lamichhaney S., Han F., Berglund J., Wang C., Almén M.S., Webster M.T., Grant, B.R., Grant, P.R., Andersson L. 2016. A beak size locus in Darwin's finches facilitated character displacement during a drought. Science 6284, 470-474

Leitch I.J., Kahandawala I., Suda J., Hanson, L., Ingrouille M.J., Chase M.W., Fay M.F. 2009. Genome size diversity in orchids: consequences and evolution. Annals of Botany 104, 469-481

Lisch D. 2013. How important are transposons for plant evolution? Nature Review Genetics 14, 49-61 
Litsios G., Sims C.A., Wüest R.O., Pearman P.B., Zimmerman N.E., Salamin N. 2012. Mutualism with sea anemones triggered the adaptive radiation of clownfishes, BMC Evolutionary Biology 12, 212 Loiseau O., Olivares I., Paris M., de La Harpe M.-L., Weigand A., Koubínova D., Rolland J., Bacon C.D., Balslev H., Borchsenius F., Cano A., Couvreur L.P., Delnatte C., Fardin F., Gayot M., Mejía F. MotaMachado T., Perret M., Roncal J., Sanin M.-J., Stauffer F., Lexer C., Kessler M. Salamin N. 2019. Targeted capture of hundreds of nuclear genes unravels phylogenetic relatinonships of the diverse Neotropical palm tribe Geonomateae. Frontiers in Plant Sciences, 884

Lynn S.K., Cnaani J., Papaj D.R. 2005. Peak shift discrimination learning as a mechanism of signal evolution. Evolution 59, 1300-1305

Machaka-Houri N., Houri A., Knio K.M., Westbury D.B. 2018. Ecological interactions of the sexually deceptive orchid Orchis galilaea. Journal of Plant Interactions 13, 315-320

Mant J., Peakall R., Schiestl F.P. 2005. Does selection on floral odor promote differentiation among populations and species of the sexually deceptive orchid genus Ophrys? Evolution 59, 1449-1463

Marques D.A., Meier J.I., Seehausen O. 2019. A combinatorial view on speciation and adaptive radiation. Trends in Ecology and Evolution, in press

McCormick M.K., Jacquemyn H. 2014. What constrains the distribution of orchid populations? New Phytologist 292, 392-400

Nazarov V., Gerlach G. 1997. The potential seed productivity of orchid flowers and peculiarities of their pollination systems. Lindleyana 12, 188-204

Neiland M.R.M., Wilcock C.C. 1995. Maximisation of reproductive success by European Orchidaceae under conditions of infrequent pollination. Protoplasma 187, 39-48

Neiland M.R.M., Wilcock C.C. 1998. Fruit set, nectar reward, and rarity in the Orchidaceae. American Journal of Botany 85, 1657-1671

Nilsson L.A. 1992. Orchid pollination biology. Trends in Ecology and Evolution 7, 255-259

Parchman T.L., Benkmans, C.W., Britch S.C. 2006. Patterns of genetic variation in the adaptive radiation of New World crossbills (Aves: Loxia). Molecular Ecology 15, 1873-1887

Parchman T.L., Buerkle, C.A., Soria-Carasco V., Benkmans, C.W. 2016. Genome divergence and diversification within a geographic mosaic of coevolution. Molecular Ecology, 25, 5705-5718

Parker G.A., Courtney S.P. 1983. Seasonal incidence: adaptive variation in the timing of life history stages. Journal of theoretical Biology 105, 147-155

Paulus H.F. 2006. Deceived males - Pollination biology of the Mediterranean orchid genus Ophrys (Orchidaceae). Journal Europäischer Orchideen 38, 303-353

Paulus H.F. 2017. Bestäubungsbiologie Ophrys in Nordspanien. Journal Europäischer Orchideen 49, 427-471

Paulus H.F. 2018. Pollinators as isolation mechanisms: field observations and field experiments regarding specificity of pollinator attraction in the genus Ophrys (Orchidacea). Entomologia Generalis 37, 261-316

Paulus H.F., Gack C. 1990. Pollinators as prepollinating isolation factors: evolution and speciation in Ophrys (Orchidaceae). Israel Journal of Botany39, 43-79

Paxton R.J. 2005. Male mating behaviour and mating systems of bees: an overview. Apidologie 36, 145-156

Peakall R. 1990. Responses of male Zaspilothynnus trilobatus Turner wasps to females and the sexually deceptive orchid it pollinates. Functional Ecology 4, 159-167 
Perrin N., Mazalov V. 1999. Dispersal and inbreeding avoidance. American Naturalist 154, 282-292

Pillon Y., Chase M.W. 2007. Taxonomic exaggeration and its effects on orchid conservation. Conservation Biology 21, 263-265

Pouyanne, M.A. 1917. La fécondation des Ophrys par les insectes. Bulletin de la Société d'Histoire Naturelle d'Afrique du Nord 43, 53-62

Pusey A., Wolf M. 1996. Inbreeding avoidance in animals. Trends in Ecology and Evolution 11, 201206

Raguso R.A. 2008. Wake up and smell the roses: the ecology and evolution of floral scent. Annual Review of Ecology, Evolution and Systematics 39, 549-569

Rakosy D., Streinzer M., Paulus H.F., Spaethe J. 2012. Floral visual signal increases reproductive success in a sexually deceptive orchid. Arthropod-Plant Interactions 6, 671-681

Rakosy D., Cuervo M., Paulus H.F., Ayasse M. 2017. Looks matter: changes in flower form affect pollination effectiveness in a sexually deceptive orchid. Journal of Evolutionary Biology 30, 19781993

Rasmont P., Haubruge E., 2014. Atlas of Hymenoptera. Université de Mons, Gembloux Agro Bio Tech, Mons, Gembloux

Rasmussen H.N., Dixon K.D., Jersakova J., Tesitelova T. 2015. Germination and seedling establishment in orchids: a complex of requirements. Annals of Botany 116, 391-402

Robbit K.M., Roberts D.L., Hutchings M.J., Davy 2014 A.J. Potential disruption of pollination in a sexually deceptive orchid by climatic change. Current Biology 24, 2845-2849

Roma L., Cozzolino S., Schlüter P.M., Scopece G., Caffaso D. 2018 The complete plastid genomes of Ophrys iricolor and $O$. sphegodes (Orchidaceae) and comparative analyses with other orchids. PLoS One 13(9):e204174.

Salisbury E. 1975. The survival value of modes of dispersal. Proceedings of the Royal Society Series B, Biological Sciences 188, 183-188

Schatz B., Geoffroy A., Dainat B., Bessiere J.M., Buatois B., Hossaert-McKey M., Selosse M.A. 2010. A case study of modified interactions with symbionts in a hybrid Mediterranean orchid. American Journal of Botany, 97, 1278-1288

Schatz B. 2017. Les orchidées de l'île de Cavallo (archipel des Lavezzi, Corse) : une surprenante abondance de l'espèce protégée Gennaria diphylla. Ecologia Mediterranea 43, 159-170

Schatz B., Sauvion N., Kjellberg F., Nel A. 2017. Plant-insect interactions: a palaeontological and an evolutionary perspective. In N. Sauvion, D. Thiéry, P.-A. Calatayud (Eds.), Insect-Plant interactions in a Crop Protection Perspective, Elsevier, The Hague, 1-24

Schiestl F.P. 2005. On the success of a swindle: pollination by deception in orchids.

Naturwissenschaften 92, 255-264

Schiestl F.P., Ayasse M. 2000. Post-mating odor in females of the solitary bee, Andrena nigroaenea (Apoidea, Andrenidae), inhibits male mating behavior. Behavioral Ecology and Sociobiology 48, 303307

Schiestl F. P., Ayasse, M. 2001. Post-pollination emission of a repellent compound in a sexually deceptive orchid: a new mechanism for maximising reproductive success? Oecologia 126, 531-534

Schiestl F.P., Ayasse M. 2002. Do changes in floral odor cause speciation in sexually deceptive orchids? Plant Systematics and Evolution 234, 111-119 
Schiestl F.P., Marion-Poll F. 2002. Detection of physiologically active flower organics using gas chromatography coupled with electroantennography. In J.F. Kackson, H.F. Linskens (Eds.), Analysis of Taste and Aroma, Springer, Berlin

Schiestl F.P., Ayasse M., Paulus H.F., Erdmann D., Francke W. 1997. Variation of floral scent emission and post pollination changes in individual flowers of Ophrys sphegodes subsp. sphegodes (Miller). Journal of Chemical Ecology 23, 2881-2895

Schiestl F.P., Ayasse M., Paulus H.F., Löfstedt C., Hansson B.S., Ibarra F., Francke W. 1999. Orchid pollination by sexual swindle. Nature 399, 421-422

Schiestl F.P., Ayasse M., Paulus H.F., Löfstedt C., Hansson B.S., Ibarra F., Francke W. 2000. Sex pheromone mimicry in the early spider orchid (Ophrys sphegodes): patterns of hydrocarbons as the key mechanism for pollination by sexual deception. Journal of Comparative Physiology A 186, 567574

Schluter D. 2000. Ecological character displacement in adaptive radiation. American Naturalist 156, S4-S16

Schlüter P.M., Schiestl F.P. 2008. Molecular mechanisms of floral mimicry in orchids. Trends in Plant Science 13, 228-235

Schlüter P.M., Xu S., Gagliardini V., Whittle E., Shanklin J., Grossniklaus U., Schiestl F.P. 2011. Stearoyl-acyl carrier protein desaturases are associated with floral isolation in sexually deceptive orchids. Proceedings of the National Academy of Sciences 108, 5696-5701

Schoener T.W. 2011. The newest synthesis: understanding the interplay of evolutionary and ecological dynamics. Science, 331 426-429

Schoenfelder K.P., Fox D.T. 2015. The expanding implications of polyploidy. The Journal of Cellular Biology 209, 485-491

Scopece G., Musacchio A., Widmer A., Cozzolino S. 2007. Patterns of reproductive isolation in mediterranean deceptive orchids. Evolution 61, 2623-2642

Scoepece G., Schiestls, F.P., Cozzolino, S. 2015. Pollen transfer efficiency and its effect on inflorescence size in deceptive pollination strategies. Plant Biology 17, 545-550

Sedeek K.E.M., Scopece G., Staedler Y.M., Schönenberger J., Cozzolino S., Schiestl F.P., Schlüter P.M. 2014. Genic rather than genome-wide differences between sexually deceptive Ophrys orchids with different pollinators. Molecular Ecology 23, 6192-6205.

Sedeek K.E.M., Whittle E., Guthörl D., Grossniklaus U., Shanklin J., Schlüter P.M. 2016. Amino acid change in an orchid desaturase enables mimicry of the pollinator's sex pheromone. Current Biology 26, 1505-1511

Seidl M.F., Thomma B.P.H.J. 2017. Transposable elements direct the coevolution between plants and microbes. Trends in Genetics 33, 842-851

Simões M., Breitkreuz L., Alvarado M., Baca S., Cooper J.C., Heins L., Herzog K., Lieberman B.S. 2016. The evolving theory of evolutionary radiations. Trends in Ecology and Evolution 31, 27-34

Sletvold N., Grindeland J. M., Ågren J. 2010. Pollinator-mediated selection on floral display, spur and flowering length phenology in the deceptive orchid Dactylorhiza lapponica. New Phytologist 188, 385-392

Sletvold N., Trunschke J., Smit M., Verbeek J., Ågren J. 2016. Strong pollinator mediated selection for increased flower brightness and contrast in a deceptive orchid. Evolution 70, 716-724

Smith B.H., Ayasse M. 1987. Kin-based male mating preferences in two species of halictine bee. Behavioral Ecology and Sociobiology 20, 313-318 
Solé RV., Sardanayés J. 2014. Red Queen coevolution on fitness landscapes. In H. Richter, Engelbrecht (Eds.). Recent Advances in the Theory and Application of Fitness Landscapes, Springer, Heidelberg, 301-338

Soltis D.E., Soltis P.S., Pires J.C., Kovarik A., Tate J. 2004. Recent and recurrent polyploidy in Tragopogon (Asteraceae): cytogenetic, genomic and genetic comparisons. Biological Journal of the Linnean Society 82, 485-501

Sonkoly J., Vojtko A.E., Tökölyi J., Török P., Sramko G., Illyés Z., Molnar V. Z. 2016. Higher seed number compensates for lower fruit set in deceptive orchids. Journal of Ecology 104, 343-351

Souche R. 2007. Ophrys insectifera complex; Ophrys subinsectifera from Spain. A new mechanism of pollination through sexual deception. Caesiana 28, 121-126

Spaethe J., Moser W. H., Paulus H. F. 2007. Increase of pollinator attraction by means of a visual signal in the sexually deceptive orchid, Ophrys heldreichii (Orchidaceae). Plant Systematics and Evolution 264, 31-40

Spaethe J., Streinzer M., Paulus H.F. 2010. Why sexually deceptive orchids have colored flowers? Communicative \& Integrative Biology 3, 139-141

Sramkó G., Gulyás G., Molnár V.A. 2011. Convergent evolution in Ophrys kotschyi (Orchidaceae) revisited: a study using nrITS and cpIGS sequences. Annales Botanici Fennici 48, 97-107

Stejskal K., Streinzer M., Dyer A., Paulus H.F., Spaethe J. 2015. Functional significance of labellum pattern variation in a sexually deceptive orchid (Ophrys heldreichii): evidence of individual signature learning effects. PLoS One 10, e0142971

Stökl J., Paulus H., Dafni A., Schulz C., Francke W., Ayasse M. 2005. Pollinator attracting odour signals in sexually deceptive orchids of the Ophrys fusca group. Plant Systematics and Evolution 254, 105120

Stökl J., Twele R., Erdmann D. H., Francke W., Ayasse, M. 2007. Comparison of the flower scent of the sexually deceptive orchid Ophrys iricolor and the female sex pheromone of its pollinator Andrena morio. Chemoecology 17, 231-233

Stökl J., Schlüter P. M., Stuessy T. F., Paulus H. F., Assu, G., Ayasse M. 2008. Scent variation and hybridization cause the displacement of a sexually deceptive orchid species. American Journal of Botany 95, 472-481

Stökl J., Schlüter P. M., Stuessy T. F., Paulus H. F., Fraberger R., Erdmann D., Ayasse M. 2009. Speciation in sexually deceptive orchids: pollinator-driven selection maintains discrete odour phenotypes in hybridizing species. Biological Journal of the Linnean Society 98, 439-451

Streinzer M., Paulus H. F., Spaethe J. 2009. Floral colour signal increases short range detectability of a sexually deceptive orchid to its bee pollinator. Journal of Experimental Biology 212, 1365-1370.

Strouds J.T., Losos J.B. 2016. Ecological opportunity and adaptive radiation. Annuals Reviews of Ecology, Evolution and Systematics 47, 507-532

Svanbäck R., Bolnick D.I. 2007. Intraspecific competition drives increased resource use diversity within a natural population. Proceedings of the Royal Society Series B 274, 839-844

Svensson G.P., Strandh M., Löfstedt. 2014. Movements in the olfactory landscape. In: L.-A. Hansson \& S. Åkesson (Eds.), Animal Movements Across Scales, Oxford University Press, Oxford, 195-218

ten Cate C., Rowe C. 2007. Biases in signal evolution: Learning makes a difference. Trends in Ecology and Evolution 22, 380-387

Thompson, J.N. 2016. Coevolution, local adaptation, and ecological speciation. Molecular Ecology 25, 5608-5610 
Triponez Y., Arrigo N., Pellissier L., Schatz B., Alvarez N. 2013. Morphological, ecological and genetic aspects associated with endemism in the Fly Orchid group. Molecular Ecology 22, 1431-1446

Tyteca D., Baguette M. 2017. Ophrys (Orchidaceae) systematics - when molecular phylogenetics, morphology and biology reconcile. Berichte aus den Arbeitskreisen Heimische Orchideen, 34, 37-103

van der Niet T., Johnson S.D 2012. Phylogenetic evidence for pollinator driven diversification of angiosperms. Trend in Ecology and Evolution 27, 353-361

Vandewoestijne, S., Róis, A.S., Caperta, A., Baguette, M., Tyteca D. 2009. Effects of individual and population parameters on reproductive success in three sexually deceptive orchid species. Plant Biology 11, 454-463

Vereecken N.J. 2009. Deceptive behaviour in plants. I. Pollination by sexual deception in orchids: a host-parasite perspective. In: F. Baluska (Ed.) Plant-environment interactions - from sensory plant biology to active behaviour, Springer Verlag, Berlin, 203-222.

Vereecken N.J., Mant J., Schiestl F.P. 2007. Population differentiation in female sex pheromone and male preferences in a solitary bee. Behavioral Ecology and Sociobiology 61, 811-821

Vereecken N.J., Schiestl F.P. 2008. The evolution of imperfect floral mimicry. Proceedings of the National Academy of Sciences 105, 7484-7488

Vereecken N. J., Schiestl, F. P. 2009. On the roles of colour and scent in a specialized floral mimicry system. Annals of Botany 104, 1077-1084

Vereecken N.J., Cozzolino S., Schiestl F.P. 2010. Hybrid floral scent novelty drives pollinator shift in sexually deceptive orchids. BMC Evolutionary Biology 10, 103

Vereecken N.J., Streinzer M., Ayasse M., Spaethe J., Paulus H.F., Stökl J., Cortis P., Schiestl F.P. 2011. Integrating past and present studies on Ophrys pollination - a comment on Bradshaw et al. Botanical Journal of the Linnean Society, 165, 329-335

Vereecken N.J., Wilson C.A., Hötling, S., Schulz S., Banketov, S., Mardulyn P. 2012. Pre-adaptations and the evolution of pollination by sexual deception: Cope's rule of specialization revisited.

Proceedings of the Royal Society Series B 279, 4786-4794

Wcislo W.T. 1987.The role of learning in the mating biology of a sweat bee Lasioglossum zephyrum (Hymenoptera: Halictidae). Behavioral Ecology and Sociobiology 20, 179-185

Wcislo W.T. 1992. Attraction and learning in mate-finding by solitary bees, Lasioglossum (Dialictus) figueresi Wcislo and Nomia triangulifera Vachal (Hymenoptera: Halictidae). Behavioral Ecology and Sociobiology 31, 139-148

Wells T.C.E., Cox R. 1991. Demographic and biological studies on Ophrys apifera: some results from a 10 year study. In T.C.E. Wells, J.A. Willems (Eds.), Population Ecology of Terrestrial Orchids, SPB Academic Publishing, The Hague, 47-61

White J., Son Y., Park Y. 2009. Temperature-dependent emergence of Osmia cornifrons (Hymenoptera: Megachilidae) adults. Journal of Economic Entomology 102, 2026-2032

Wiklund C., Fagerström T. 1977. Why do males emerge before females? A hypothesis to explain the incidence of protandry in butterflies. Oecologia 31, 153-158

Willems J.H. 1994. Bottle-necks in establishment and survival of small populations of orchids in Western Europe. In: P. Brederoo, D.W. Kapteyn den Boumeester (Eds.), Euroorchis 92, Proceedings of the International Symposium on European Orchids, Stichting Uitgeverij Koninklijke Nederlandse Natuurhistorische Vereniging, Utrecht, 72-82 
Williams R., Edwards M. 2012. Bees in Britain. BWARS bee-book-section 4, http://www.bwars.com/sites/www.bwars.com/files/diary downloads/Britain\%27s Bees Table of S pecies.pdf

Wilson E.O. 1992. The diversity of life. Harvard University Press, Massachussets

Winston M.L. 1991. The biology of the honey bee. Harvard University Press, Cambridge, Massachusetts

Wu C.I. 2001. The genic view of the process of speciation. Journal of Evolutionary Biology 14, 851-865

Wu C.I., Ting C.T. 2004. Genes and speciation. Nature Reviews Genetics 5, 114-122

Xu S., Schlüter P.M. 2015. Modeling the two-locus architecture of divergent pollinator adaptation: how variation in SAD paralogs affects fitness and evolutionary divergence in sexually deceptive orchids. Ecology and Evolution 5, 493-502

Xu S., Schlüter P.M., Scopece G., Breitkopf H., Gross K., Cozzolino S., Schiestl F.P. 2011. Floral isolation is the main reproductive barrier among closely related sexually deceptive orchids. Evolution 65, 2606-2620

Xu S., Schlüter P.M., Grossniklaus U., Schiestl F.P. 2012. The genetic basis of pollinator adaptation in a sexually deceptive orchid. PLoS Genetics 8, e1002889

Zayed A., Packer L. 2005. Complementary sex determination substantially increases extinction proneness of haplodiploid populations. Proceedings of the National Academy of Sciences 102, 1074210746

Zhang L., Hu J., Han X., Li J., Gao Y., Richards C.M., Zhang C., Tian Y., Liu G., Gul H., Wang D., Tian Y., Yang C., Meng M., Yuan G., Kang G., Wu Y., Wang K., Zhang H., Wang D., Cong P. 2019. A high-quality apple genome assembly reveals the association of a retrotransposon and red fruit colour. Nature Communications 10, 1494.

Zonneveld C., Metz J.A. 1991. Models on butterfly protandry: virgin females are at risk to die. Theoretical Population Biology 40, 308-321 